\title{
In Vitro CSC-derived Cardiomyocytes Exhibit the Typical microRNA-mRNA Blueprint of Endogenous Cardiomyocytes
}

Mariangela Scalise

Magna Graecia University

Fabiola Marino

Magna Graecia University

Luca Salerno

Magna Graecia University

Mancuso Teresa

Magna Graecia University

Donato Cappetta

University of Campania

Antonella Barone

Magna Graecia University

Elvira Parrotta

Magna Graecia University

Annalaura Torella

University of Campania

Domenico Palumbo

University of Salerno

Pierangelo Veltri

Magna Graecia University

Antonella De Angelis

University of Campania

Liberato Berrino

University of Campania

Francesco Rossi

University of Campania

Alessandro Weisz

University of Salerno

Marcello Rota

New York Medical College

Konrad Urbanek 
Magna Graecia University

Bernardo Nadal-Ginard

Magna Graecia University

Daniele Torella ( $\sim$ dtorella@unicz.it)

Magna Graecia University https://orcid.org/0000-0002-4915-5084

Eleonora Cianflone

Magna Graecia University

\section{Article}

Keywords: CSCs, cardiomyogenic specification, cardiomyogenic differentiation, cardiomyocytes, microRNA, myo-miRs

Posted Date: June 25th, 2021

DOl: https://doi.org/10.21203/rs.3.rs-624153/v1

License: (c) (1) This work is licensed under a Creative Commons Attribution 4.0 International License. Read Full License

Version of Record: A version of this preprint was published at Communications Biology on September 30th, 2021. See the published version at https://doi.org/10.1038/s42003-021-02677-y. 


\section{Abstract}

miRNAs modulate cardiomyocyte specification in embryonic hearts and in pluripotent stem cells by targeting mRNAs of cell cycle regulators and acting in gene regulatory loops that complete commitment to the cardiac muscle lineage. It is still unknown if/to-what-extent these miRNA/mRNA networks are operative during cardiomyocyte differentiation of adult cardiac stem/progenitor cells (CSCs). Clonallyderived mouse CSCs differentiated into contracting cardiomyocytes in vitro (iCMs). RNASeq comparison of "CSCs vs. iCMs" mRNome and microRNome showed a balanced up-regulation of sarcomere and mitochondrial related mRNAs together with a down-regulation of cell cycle and DNA replication mRNAs. The down-regulation of cell cycle genes and the up-regulation of the mature myofilament genes in iCMs did not reach the levels of mouse terminally differentiated adult cardiomyocytes (aCMs), while they get to intermediate levels between those of fetal and neonatal cardiomyocytes. Cardiomyo-miRs were upregulated in iCMs while those miRs positively regulating stem cell expansion and self-renewal were downregulated. The specific networks of miRNA/mRNAs operative in iCMs closely resembled miRNA/mRNA networks of aCMs. Two of these miRs, miR-1 and miR-499, enhanced myogenic commitment toward terminal differentiation of iCMs. In conclusions, CSC specification/differentiation into contracting iCMs follows known cardiomyo-MiR-dependent developmental cardiomyocyte differentiation trajectories and iCMs transcriptome/miRNome resembles that of aCMs.

\section{Introduction}

Cardiomyocyte (CM) specification, differentiation and maturation are complex processes that require the activity of many temporally and spatially modulated gene regulatory networks ${ }^{1-3}$. microRNAs (miRs) play a major role regulating these cardiac transcriptional pathways which drive CM development, maturation and function ${ }^{4-8}$. The relevance of miRNAs in cardiac development and function is documented by the effect of cardiac-restricted knockout of Dicer, the gene encoding RNase III endonuclease essential for normal miR processing ${ }^{9-11}$. Cardiac progenitor-specific deletion of Dicer using a CRE recombinase under the control of the endogenous Nkx2.5 promoter at E8.5, led to embryonic lethality at E12.5 $5^{9,10}$. Inactivation of Dicer using cTNT-Cre led to embryonic lethality at E15.5 ${ }^{11}$. Finally, deletion of Dicer by $a-\mathrm{MHC}$-Cre caused death four days after birth by heart failure ${ }^{9}$.

In vitro differentiation of embryonic stem cells (ESCs), as well as of induced pluripotent stem cells (iPSCs), into CMs recapitulate the CM development, differentiation and maturation in the embryonic life $\mathrm{e}^{12-14}$. These in vitro iPSC-based model systems have revealed different roles played by specific miRs in cardiomyocyte differentiation. miR-1, the most abundant miR in CMs, functions cooperatively with miR133 to promote mesoderm formation and to suppress non-muscle gene expression in ESCs ${ }^{15,16}$. miR199a-3p, miR-214-3p, miR-483-3p and miR-322/-503 were shown to be relevant for mesodermal specification of mesodermal cardiac progenitor cells ${ }^{16,17}$. miR-208a, $-208 \mathrm{~b}$, and -499 are intronic miRs encoded by the Myh6, Myh7, and Myh7b genes, respectively. Because their expression depends on the expression of the respective myosin genes, they were named myo-miRs ${ }^{18}$. A combination of miR-1, miR- 
133 , miR-208, and miR-499 was able to directly induce the in vitro cellular reprogramming of fibroblasts into $\mathrm{CMs}^{15-21}$. Combination of miR-125 b-5p, -199a-5p, -221, and - 222 expression into ESC-derived CMs improved their maturation ${ }^{22}$. Let-7, a non-cardiac-specific miR, is required to enhance a number of functional properties relevant to $\mathrm{CM}$ maturation by promoting metabolic transition from glucose to fatty acids $^{23}$. Despite these findings, ESC- or iPSC-derived in vitro CMs are not fully differentiated and continue to reveal immature properties of embryonic/fetal $\mathrm{CMs}^{12,24,25}$.

The adult cardiac stem/progenitor cells (CSCs) are a small cohort of cardiac tissue resident progenitor cells present in the postnatal mammalian heart ${ }^{26-43}$. CSCs can be cloned, propagated in long-term culture and maintained in an undifferentiated self-renewing state; when grown in suspension they form spheres similar to pseudo-embryoid bodies called cardiospheres (CS ${ }^{44-46}$. CSCs grown in differentiation media for endothelial (EC), smooth muscle (SMC), and cardiomyocyte (CM) lineages, they acquire phenotypic characteristics of these different cell types ${ }^{44-46}$. CSC transplantation as well as their in-situ activation were shown to differentiate into new myocytes, microvasculature and fibroblasts in response to ischemic and non-ischemic myocardial damage ${ }^{36-39,47-54}$. Despite the above data, several genetic cell fate mapping studies failed to show a significant contribution of the endogenous CSCs to new CM formation in adulthood and after injury ${ }^{55-60}$. While we have challenged these results and shown that the significant limitation of the cell fate mapping approaches used in these studies preclude mapping the fate and the role of the CSCs in the in vivo CM renewal $43,48,49,53$, from a miRNA/mRNA network perspective it is still unknown, to what extent the miRNA/mRNA profile of the CSC-derived CMs overlaps that of the adult CMs. It is also unknown whether the differentiation of CSCs into CMs undergoes the transcriptional switch typical of cell cycle exit during cardiac myocyte differentiation up-regulating known myo-miRs and more generally the set of more abundant miRs in adult CMs. The major objective of this study is to fill this gap while defining the level of maturation of the in vitro CSC-derived CMs.

\section{Results}

\section{Adult CSCs in vitro differentiate into spontaneously contractile cardiomyocytes closely resembling fetal to neonatal cardiomyocytes}

Heart development from the embryonic cardiac mesoderm requires the spatially and temporally regulated expression of specific growth factors, such as Wingless/WNT (WNTs), Bone morphogenetic proteins (BMP) and Nodal/Activin molecules followed by the ensuing activation of their dependent gene cascades. These specific cardiac morphogens are also necessary and sufficient to specify and differentiate ESCs and CSCs into CMs in defined conditions in vitro $29,36,38$.

A cardiomyogenic differentiation protocol initially developed for adult rat primary and clonal CSC-derived cardiospheres (CS ${ }^{29}$, similar to a protocol to generate cardiomyocytes from cardiovascular progenitors (CPC) from ESC-derived embryoid bodies $(E B)^{61}$, has been used here to efficiently differentiate mouse CSCs into functional beating CMs. Cloned CSCs $\left(1 \times 10^{5}\right)$ were placed for 4 days in bacteriological dishes 
containing CSC growth medium to generate $\mathrm{CSs}^{36,48}$. The CSs were then switched to base differentiation medium (StemPro34) containing BMP-4 (10ng/ml), Activin A (50ng/ml), basic fibroblast growth factor ( $\beta$ FGF) $(10 \mathrm{ng} / \mathrm{ml})$, WNT-11 $(150 \mathrm{ng} / \mathrm{ml})$ and WNT-5a $(150 \mathrm{ng} / \mathrm{ml})$ from day 4 to 8 with complete media replaced every 48 hours (see Fig. 1A-C). Differentiating CSs were then transferred to laminin-coated dishes in StemPro34 medium with Dickkopf-related protein (DKK-1) $(150 \mathrm{ng} / \mathrm{ml})$ added every 48 hours together with $50 \%$ fresh medium replacement for up to 14 days to inhibit the canonical Wnt/ $\beta$-catenin pathway, an inhibition shown to be required for cardiomyocyte differentiation during heart development ${ }^{36-41}$. The differentiating CSCs start spontaneous contractile activity and rhythmic beating at day 10 (i.e., two days after the start of DKK-1 administration) (Supplementary Movie 1).

In order to evaluate the structural characteristics of these CSCs-derived beating cells, immunocytochemistry with antibodies directed against myofilament proteins, such as cardiac Troponin I (cTnl) and myosin heavy chain (MF20 antibody) was carried out. As shown in Fig. 1E,F, both sarcomeric proteins, cTNNI and MHC, were expressed in high percentages, respectively $75 \pm 11 \%$ and $83 \pm 7 \%$, of CSCderived CMs (hereafter identified as CSC-induced CMs, iCMs) of beating spheres at 10 day (Fig. 1D-F). In particular, dissociated iCMs at 14 days in differentiation medium showed a well-developed sarcomeric striations that more closely resembled neonatal cardiomyocytes (neoCMs isolated from 2 days old mice) which are still immature compared with that of adult terminally differentiated CMs (aCMs, isolated from 10 weeks old mice) (Fig. 1G). RT-PCR analysis shows that at the end of the differentiation protocol the main cardiac transcription factors, Gata-4, Nkx2.5, Mef2C and Hand2 were robustly up-regulated with concurrent expression of the contractile protein genes, such as cTnnt2, Myh7 and Actc1, (Fig. 1H). Importantly, to assess the level of maturation of iCMs, we compared mRNA levels of key cardiac transcription factors and myofilament proteins of iCMs with whole fetal mouse heart from 11.5 embryonic day (for simplicity abbreviated as E11.5 Heart), neoCMs and aCMs. As previously reported ${ }^{62}$, mRNA levels of cardiac developmental genes, such as Nkx2.5, Gata-4, and Hand2 showed highest expression in iCMs (Fig. 2A,B). On the other hand, all myofilament protein genes in iCMs reached an overall expression level intermediate between fetal and neonatal $\mathrm{CMs}$ but that still is significantly lower than aCMs (Fig. 2A,B).

Overall, the cytological, immunofluorescence and gene expression analyses indicate that clonal adult mouse CSCs differentiate into spontaneously beating contractile cells with the structural and biochemical phenotype of immature fetal/neonatal cardiomyocytes.

Global transcriptome of CSC-derived iCMs shows a gene expression pattern of well differentiated yet immature cardiomyocyte phenotype

To better evaluate the transcriptional phenotype of the mouse iCMs, their global transcriptome after 14 days in differentiating media was obtained by RNASeq analysis and compared to that of their undifferentiated progenitors, the CSCs ( $n=3$ biological replicates for both iCMs and CSCs). To assess the iCMs broad cardiac muscle phenotype and the extent of their cardiomyocyte differentiation, their transcriptome was compared with that of mature adult cardiomyocytes (aCMs) isolated from 10-week 
old mice ( $\mathrm{n}=2$ biological replicates). Enrichment Analysis (EA) was used to identify the up- and downregulated gene sets grouped by the Gene Ontology resource tools ${ }^{63}$ in the different samples.

Comparison of the differential gene expression of the "iCMs vs. CSCs" (Fig. 3A) as grouped by the Gene Ontology tools (Fig. 3B) revealed that 121 mRNAs were down-regulated while 2601 mRNAs were upregulated in iCMs (Fig. 3A). CSCs are highly enriched with genes involved with DNA replication, cell cycle, telomere maintenance and epigenetic regulation of gene expression which were down-regulated in iCMs (Fig. 2B). The up-regulated gene sets in iCMs are involved in myogenic cell differentiation, heart generation, BMP signaling modulating mesodermal specification and differentiation, positive regulation of metabolic processes, signal transduction and cytokine production and secretion (Fig. 3B,D).

Conversely, the EA of the "iCMs vs. aCMs" (Fig. 3C) as grouped by the Gene Ontology tools (Fig. 3D) revealed that 944 mRNAs were significantly lower while 1915 mRNAs were significantly higher in iCMs compared to aCMs (Fig. 3C). The genes having the highest expression levels in the aCMs are mainly involved with cardiac muscle contraction, cell action potential and calcium regulation (Fig. 3D). On the other hand, the genes that are more highly expressed in iCMs are involved with cellular and metabolic processes and signal transduction (Fig. 3D). Furthermore, even if with less pronounced difference, among the mRNAs more highly expressed iCMs were those involved with cell cycle competence (Fig. 3D).

Finally, we built a list of 176 myocyte genes most expressed in the aCMs (that for simplicity we grouped under the name of 'myogenes' to signal their high enrichment in the mature aCMs) (Table S1)A heatmap of their expression in CSCs, iCMs and aCMs was generated (Fig. 3E). Compared to CSCs, the most upregulated genes in iCMs were cardiac-specific transcription factors, growth factors, growth factor receptors and their main downstream signaling molecules (Mef2a, Mef2c, Gata4, Nkx2.5, Ryr2, Tbx-2, Tbx-3, Tbx-5, Tbx-20, Myocd, Wt1, SRF, Notch1, Bmp10, Wnt10b, Cxcr4, Fgfr2, Tgfbr1, Tgfbr2, Tgfbr3, and Smad1) known to be essentials for cardiac cell commitment and differentiation during fetal development ${ }^{64-66}$ (Fig. 3E). As expected for a population of cells committing toward a terminally differentiated lineage such as adult cardiomyocytes, iCMs show a major reduction of cell cycle genes like Cdkn2a and Cdca3 (Fig. 3E).

It is well known that the pocket protein family of tumor suppressors, and Rb specifically, are crucial for cell cycle withdrawal, maturation and maintenance of the terminally differentiated state of $\mathrm{CMs}^{67,68}$. In agreement with previous data ${ }^{69}, \mathrm{Rb}$ (also known as mature $\mathrm{Rb}$ or $\mathrm{Rb} 1$ ) mRNA is scant or undetectable in E11.5 Hearts, but it is up-regulated in neoCMs, and it becomes the predominant pocket protein in adult CMs (Supplementary Fig. 1). In contrast, p107 (also known as Rb like 1or Rbl1) mRNA expression is reciprocal to that of Rb being highest in E11.5 Hearts and lowest in the aCMs (Supplementary Fig. 1). p130 (also known as Rb like 2 or Rbl2) mRNA expression peaks in neoCMs and is subsequently downregulated becoming very low in aCMs (Supplementary Fig. 1). This Rb pocket protein transition is necessary for aCM terminal differentiation ${ }^{67,68}$. Of note, iCMs significantly down-regulate cyclin A2 mRNA (Cnna2) compared to CSCs while Rb1 is up-regulated and Rbl1 is down-regulated (Fig. 3F). Accordingly, $\mathrm{Rbl} 2$ is up-regulated in iCMs compared to CSCs (Fig. 3F). The pattern of pocket protein gene expression 
further suggests that the differentiation and commitment of CSC-derived iCMs regulates the cell cycle machinery in a manner similar to the one occurring in the transition from proliferating cardiomyocytes to adult terminally differentiated CMs which occurs in the early post-natal period ${ }^{67,68}$.

The adult CM-specific gene signature set, the myogenes, and particularly the sarcomere-related genes (i.e. Actc1, Ttn, Tnnt2, Myh6, Myh7, Myh11, Myo18b, Myl2) were up-regulated in iCMs vs. the CSCs and further up-regulated in the aCMs (Fig. $3 \mathrm{E}$ ). Considering the immature state of $\mathrm{iCMs}^{36}$, it is not surprising that the adult CM-specific gene signature it is not as highly expressed in the iCMs as in the aCMs (Fig. 3E). Also, because in the iCM population not all the cells are differentiated to the same degree (see above), the myogene set is diluted while the cell cycle genes are not totally down-regulated in iCMs compared to aCMs (Fig. 3E).

Overall, these data show that CSCs, when properly induced in vitro, are robustly cardiomyogenic through the activation of the entire gene network characteristic of the adult cardiomyocyte phenotype. Nevertheless, the in vitro CSC-derived CMs, even though spontaneously contracting, still have an immature cardiac muscle phenotype, closely resembling fetal/neonatal CMs which most are likely terminally withdrawn from the cell cycle a fraction of cells maintain a limited proliferative potential while lacking the levels of expression of the sarcomeric genes typical of the adult terminally differentiated phenotype.

\section{miRNome of CSC-derived CMs recapitulates miRNome of adult cardiomyocytes}

MiRNAs, functioning mainly as tuners and providers of robustness of mRNA expression ${ }^{70}$, significantly contribute to cardiomyocyte specification/differentiation and heart development. We assessed the miRNA expression profile obtained by RNASeq from the same samples used for the mRNA transcriptome analysis. To identify miRNA sequences, the reads were mapped to the mouse genome and aligned to miRBase. Based on the high-throughput sequencing results, we used a hierarchical clustering algorithm to analyze differentially expressed miRNAs in the respective biological replicates of the three cell populations: CSCs, iCMs and aCMs. While consistent expression patterns are observed in the miRNA heatmaps intra-group, several differences among the three biological samples were evident (Fig. 4A). To characterize these differences we performed a k-means clustering which separated miRNAs into 3 cluster groups (Fig. 4B). Cluster 1 is the group of miRNAs whose expression is significantly higher in aCMs compared to the CSCs and iCMs (Fig. 4B). Panther Classification revealed that this miRNA Cluster 1 consists of a group of miRs (listed in Table S2) that putatively target mRNAs involved with cardiac ventricle formation and cardiac chamber formation. Cluster 2 is a group of miRNAs (see Table S3) that are significantly higher expressed in CSCs compared to the iCMs and aCMs (Fig. 4B) and that putatively target mRNAs involved with cell proliferation. Finally, the group organized as cluster 3 (Table S4) shows miRNAs up-regulated in iCMs compared to CSCs and aCMs (Fig. 4B) that putatively target mRNAs involved with positive stem cell population maintenance. A Principal Component Analysis (PCA) of global miRNA expression in the three cell populations locate the miRNA profile of CSCs and aCMs at opposite poles while iCMs occupy an intermediate position as expected for a cell intermediate between a 
progenitor and its mature progeny (Fig. 4C). The same difference among the three cell populations were also reproduced in a distance heatmap (Fig. 4D).

Volcano plots of pairwise comparison of miRNA expression between cell populations (Supplementary Fig. 2A-C) were generated. In the iCMs vs. CSCs comparison, when considering as significant a fold change of [1.5], out of a total 1652 miRNAs, 184 were down-regulated and 283 were up-regulated in the iCMs (Fig. 4E and Supplementary Fig. 2A, Table S5). These down-regulated and up-regulated miRNAs in the iCMs are related to biological processes involved with heart development, cell differentiation, calcium ion transport, cardiac tissue development and heart contraction (Fig. 4F) ${ }^{71-75}$. On the other hand, in "iCMs vs. aCMs" comparison, out of a total 1652 miRNAs, 175 were down-regulated and 271 were upregulated in iCMs (Fig. 4G and Supplementary Fig. 2B, Table S6). These differentially regulated miRNAs, are involved with cell proliferation, cell differentiation, embryonic heart tube development, mesoderm development, stem cell differentiation and maintenance and adult heart development (Fig. 4H) ${ }^{76-79}$.

Myogenic miRs describe a class of tissue specific and developmental stage-specific cardiac miRNAs that regulate the gene expression in cardiac muscle $e^{80,81}$. Several studies have provided evidence that myogenic miRs play an important role in differentiation and proliferation of muscle cells, mainly controlling cell proliferation/apoptosis, epigenetic remodeling, ion channel regulation, in addiction to regulation of the a-MHC to $\beta-\mathrm{MHC}$ switch and in the control of the myofiber gene program identity 80,81 . Here, the miRNAs known for their involvement with myogenesis and myogenic differentiation were combined with those most expressed in the aCMs and identified as cardiomyo-miRs (Fig. $4 \mathrm{I}$ and Supplementary Fig. 2C). Their genomic distribution is shown on Circos plot in Supplementary Fig. 2D. In particular, cardiomyo-miRs, miR-1a 3p, miR-133a-1, miR-133a-2, miR-204, miR-335, miR-486, miR-490, and miR-499, highly expressed in aCMs (Fig. 4I) were all also up-regulated in the iCMs (Fig. 4I). Of particular interest the levels of expression of the miR-208a and miR-208b, which are intronic to and encoded by Myh6 and Myh7 (myo-miRs), respectively, up-regulate similarly to the corresponding myosin genes in iCMs. Nevertheless, these miRs up-regulated in the iCMs showed expression levels significantly lower than in the aCMs (Fig. 4I,J) as visualized in the Box plot (Fig. 4J). In contrast to the cardiomyo-miRs, the miRNAs involved in positively regulating cell proliferation and self-renewal, such as miR-183, miR-182, miR-96, miR-298, miR-296-3p, and miR-296-5p, were highly expressed in CSCs in the comparison to aCMs (Fig. 4I).

Overall, these data suggest that cardiomyocytes derived from CSCs up-regulate the known cardiomyomiRs, which further confirms the bona fide cardiomyogenic phenotype of these cells. Yet, in consonance with the immature phenotype of the iCMs, most of these miRs have an expression level in these cells that is still significantly lower than in aCMs.

\section{Identification of miRNA/mRNA interactions and regulatory networks potentially modulating CSC specification and differentiation into cardiomyocytes}

The identification of putative gene targets of each miRNA is an important first step to start elucidating its potential functions. miRNAs usually act by either inducing degradation or repressing translation of their 
target mRNAs ${ }^{82,83}$. Even though individual miRNAs are associated with specific cell functions, it is clear that the miRNome simultaneously at any given time acts over multiple mRNAs encoding proteins with similar, opposed or intertwined roles in their respective gene networks ${ }^{81}$. Therefore, to identify putative mRNA targets of the differentially expressed miRNAs, we performed an integrated bioinformatics analysis of the differentially expressed miRNA and mRNA in the CSCs, iCMs and aCMs. We restricted the analysis to the validated and putative/predicted mRNA targets involved in cardiomyocyte differentiation, cell cycle, heart development, and stem cell differentiation and maintenance (Fig. 5,6 and Figures S3-S5).

Independent networks containing only known and predicted miRNA and their relative mRNA targets putatively or known to be involved in the process of cardiomyogenic specification and differentiation of CSCs were identified. Interestingly, the molecular networks built from the miRNA/mRNA targets comparison of iCMs vs. CSCs closely resembled the same networks constructed from the comparison of aCMs vs. CSCs (Figs. 5 and 6). In particular, the cardiomyocyte differentiation miRNA/mRNA network built on the down-regulated and up-regulated miRNAs (and respectively up-regulated and down-regulated mRNAs) was 80\% similar in its entire dataset in both, the "iCMs vs. CSCs" and "aCMs vs. CSCs" comparisons (Fig. 5). Similarly, the miRNA/mRNA network describing the cell cycle process was similar for 70\% in its entire dataset in the "iCMs vs. CSCs" and "aCMs vs. CSCs" comparisons (Fig. 6). As pertinent examples, miR-125b-1-3p, miR-344d-3p, miR-206-3p were down-regulated while their respective targets Nkx2.5, Tbx5, Hand2 mRNAs were up-regulated in "iCMs vs. CSCs" as well as in "aCMs vs. CSCs" comparisons (Fig. 5). In contrast, genes involved in stem cells maintenance, self-renewal and development such as Foxp1, Cited2, Lrp6, Pdgfra, Isl1 were found down-regulated when specific miRs such as miR-1a-3p, miR-499-5p, miR-34a-5p and miR-335-5p were up-regulated in "iCMs vs. CSCs" as well as in "aCMs vs. CSCs" comparisons (Fig. 5). Despite the qualitative similarities and the clear progression of CSCs toward myogenic commitment, the levels of the specific miRNAs and their putative mRNA targets in iCMs remain different from aCMs (Figs. 5 and 6).

The miRNA/mRNA network involved in the cell cycle control highlighted the down-regulation of cyclin and cyclin-dependent kinases, such as Ccnd1, Cdk6, Cdk14 and transcription factors such as E2f1, E2f3, E2f5 when miR-1a-3p, miR-499-5p, miR-34a-5p were up-regulated in both the "iCMs vs. CSCs" as well as in the "aCMs vs. CSCs" comparisons (Fig. 6). Conversely, the down-regulation of miR-96-5p and miR-34c-5p (as well as miR-34b-5p) was associated with up-regulation of genes involved in stem cells differentiation and cell cycle arrest such as Foxo4 and Notch1 (Fig. 6). The miRNA/mRNA networks describing heart development, and stem cell differentiation and maintenance showed a similar trend as the abovementioned networks (see Figure S3-S5).

Overall, despite that direct miRNA/mRNA interactions were not validated experimentally, the bioinformatics predictions outlined above supports the notion that CSC differentiation into iCMs activates cardiomyocyte-specific and cell-cycle arrest gene networks similar to those orchestrating cardiac development and maturation.

\section{miR-1 and miR-499 enhanced myogenic commitment of CSC-derived iCMs in vitro}


The above-described miRNA/mRNA networks show that miRNA profile comparison between "iCMs vs. CSCs" resembles that between "aCMs vs. CSCs". To further validate these comparisons we analyzed by RT-PCR several of the up-regulated miRNAs identified by the miRNA/mRNA networks in both "iCMs vs. CSCs" and 'aCMs vs. CSCs' comparisons (Fig. 7A). miR-1 and miR-499 were the ones to show the highest expression in aCMs and are also highly expressed in the iCMs albeit at a significantly lower level than in the aCMs (Fig. 7A). Previous data has shown that these two miRs can foster the myogenic differentiations of both ESCs and cardiovascular progenitors ${ }^{17,84}$. Thus, we tested whether the transduction of these two miRNAs, either separately or in combination, would further enhance the myogenic commitment of CSC-derived iCMs.

CSCs were transduced with lentiviral vectors encoding for miR-1, miR-499, miR-1 + miR-499 or a scrambled miR. 72 hours after the miRNA transductions, miR-1 and miR-499 levels were several folds higher when compared to the scrambled miRNA transduction (Fig. 7B). 48 hours after efficient transduction with miR-1 and/or miR-499, CSC underwent the myogenic differentiation assay as shown above (Fig. 1A). 14 days later, direct targets of these two miRNAs involved with the cell cycle, such as Cyclin D2 and Cdk6 for miR-1 and Ccna2 for miR-499 were down-regulated in iCMs transducted with the respective miRs (Fig. 7C). These data show that both, miR-1 and miR-499, negatively regulate the cell cycle gene network, which is typical of the myogenic terminal differentiation of cardiac muscle cells. Interestingly, iCMs transduced with miR-1 and/or miR-499 show a significantly higher expression of Nkx2.5, Gata-4, Mef2c, Tbx5, Hand2, Myo6, Myo7, Tnnt3, Actc1 and Atp2a when compared to scrambled miRNA transduction (Fig. 7D,E). In particular, iCMs transducted only with miR-1 up-regulated several genes such as Nkx2.5, Mef2c, Gata4, Atp2a. Transduction only with miR-499 positively regulated the expression of Tnnt3 and Actc1. Interestingly, co-transduction with miR-1 and miR-499 further promoted the expression of Tbx5, Hand2, Myo6 and Tnnt3, while the expression of Tnnt2, Myl2, Myo7, and Atp2a was not further increased when compared with the transduction with only single miRNA. The expression of Myl2, Myo7 and Tnnt2 was not affected by neither single miRs nor by their combination. From these data it is however not possible to determine whether the enhanced expression of this set of myocardialspecific genes upon the transfection of miR-1, miR-499 or both is just the indirect consequence of suppression the cell cycle genes and/or the down regulation of unknown negative regulator/suppressor mRNAs dampening the expression of muscle-specific mRNAs in the early stages of cardio-myogenic differentiation (Fig. 7B,D-E).

Overall, these data support the conclusion that the miRNA/mRNA network built by the bioinformatic analysis identified miRNAs involved in myocyte commitment and terminal differentiation as indeed miR-1 and miR-499 were able to enhance myogenic commitment of CSC-derived iCMs toward the terminal differentiation and cell cycle withdrawal typical of adult CMs.

\section{Discussion}

The principal findings of this study are as follows: (i) mouse CSC in vitro robustly differentiate into beating cardiomyocytes (iCMs); (ii) CSC-derived iCMs transcriptome and miRnome profiles recapitulate 
that of adult CMs even though mRNA and miRNA levels reflect an immature phenotype closer to fetal/neonatal CMs; (iii) CSCs differentiation into CMs is characterized by the switching on of the miRNA/mRNA networks operative in the adult CMs; (iv) two miRs from these miRNA/mRNA networks, miR-1 and miR-499, individually and together enhance myogenic commitment and terminal differentiation of CSC-derived iCMs.

From embryonic to adult life, mammalian cardiomyocytes (CMs), generated from cardiac progenitors within the mesodermal layer, undergo a differentiation and maturation process characterized, among other traits, by permanent exit from the cell cycle, development of sarcomeric structures, $\mathrm{Ca}++$ storage and release system, binucleation and increased metabolic demand. This process is transcriptionally regulated by specific myogenic mRNAs which expression is modulated by a family of miRNAs ${ }^{4}$. During embryonic development, Wnt, BMP, and Nodal/Activin pathways coordinate various transcriptional and growth factors to promote cardiac progenitor cells proliferation and differentiation. BMPs are members of the transforming growth factor-beta (TGF- $\beta$ ) super-family that play an essential role in most of the morphogenetic processes during development ${ }^{85}$. Mesodermal induction and cardiac differentiation require BMP signaling ${ }^{86,87}$. The activation of $\mathrm{Wnt} / \beta$-catenin signaling promotes cardiac differentiation, but during gastrulation inhibits heart formation ${ }^{88}$. Early in mammalian cardiac myogenesis, Wnt/ $\beta$ catenin signaling activation is indispensable for cardiac differentiation in P19 embryonic cells ${ }^{89}$ and highlight the biphasic role of $\mathrm{Wnt} / \beta$-catenin signaling in cardiomyocyte differentiation: activation is required to commit mesenchymal cells to the cardiac lineage; down-regulation of $\beta$-catenin is needed for cardiomyocyte differentiation at later stages. These important molecular pathways have been used in vitro to direct differentiation of adult CSCs into $\mathrm{CMs}^{36,90-92}$. Here we further show that a refined protocol of sequential and step-by-step administration of BMP-4, Activin A, $\beta-F G F$ and DKK-1 to clonal CSCs increase the expression of myogenic genes and the number of Troponin or Myosin positive cells, generating spontaneously beating cardiomyocytes in vitro (iCMs).

Transcriptome analysis is a powerful genomic tool that facilitates the identification of whole gene expression networks and regulatory mechanisms ${ }^{93}$. In this study, whole gene expression profiling by mRNASeq analysis together with different bioinformatic tools of CSCs and CSC-derived iCMs revealed a molecular signature in iCMs that closely resemble that of adult terminally differentiated CMs (aCMs). Indeed, the most relevant enriched pathways and functions describing the comparisons of CSCs with either iCMs or aCMs were similar and related to cell cycle progression, cardiac function and maturation.

Also, genome-wide small RNA sequencing identified candidate miRNAs involved with CSCs differentiation into iCMs. Comparison of the miRNome profiles of CSCs with those of iCMs and aCMs identified miRNAs that potentially play a role in the development of the CM phenotype. Interestingly, iCMs express a miRNA profile similar to the miRs set typical of adult CMs.

Despite the robust activation of a proper myocyte differentiation molecular and cell functional program, the quantitative expression of myogenic miRNAs and related mRNAs in the iCMs is intermediate between E11.5 fetal and neonatal cardiomyocytes ${ }^{36}$. In part, this immature phenotype of the iCMs might be due to 
the fact that we compared the bulk population of iCMs deriving from CSCs, which is highly heterogeneous in terms of the differentiation stage of the cells, with pure clonal CSCs and pure isolated aCMs. Thus, even though the differentiation protocol is robust and highly efficient we didn't compare a pure population of iCMs with pure CSCs and aCMs. Single cell mRNA and miR profiling will be required to determine the stage of differentiation the iCMs can reach in vitro.

The bioinformatic tools built miRNA/mRNA networks operative in both, iCMs and aCMs, strongly suggesting that CSC-derived iCMs are generated through a well-orchestrated miRNA/mRNA developmental networks from stemness/progenitor to CM-specific which are also operative in the aCMs and highlight potential targets to improve iCM maturation. Indeed, miR-1 and miR-499, already known for their role in the differentiation of ESCs and adult cardiac progenitors into $\mathrm{CMs}^{17}$ when up-regulated in the iCMs improved the maturation of these cells towards the typical terminal differentiation of aCMs. miR-1 and miR-499, independently and together, further reduced the expression of positive cell cycle regulatory genes, such as CyclinA2 and Cyclin D, while increasing the expression of negative cell cycle regulatory genes, such as Rb1, which are known to be crucial for $\mathrm{CM}$ terminal differentiation ${ }^{69,70,93}$. Furthermore, miR-1 and miR-499 further increased the expression of sarcomere genes in iCMs.

In conclusion, under proper in vitro culture conditions, CSCs robustly differentiate into functional beating cardiomyocytes undergoing changes in their transcriptome/miRnome which closely resembles that of adult CMs further supporting the true myogenic stem/precursor nature of these cells.

\section{Materials And Methods}

\section{Cell Culture}

Mouse Cardiac stem cells (CSCs) were isolated from adult mouse hearts by enzymatic dissociation using gentleMACS Dissociator (Miltenyi Biotec) as previously described ${ }^{36,48}$. Cloned CSCs were generated by single cell deposition into wells of 96 -well gelatin coated Terasaki plates by serial dilution ${ }^{36,48}$. Mouse CSCs clones were expanded on gelatin-coated dishes in CSCs growth medium containing of a 1:1 ratio of Neurobasal medium (Gibco, Life Technologies) and DMEM-F12Ham's (Gibco, Life Technologies) implemented with insulin-transferrin-selenium (1\%, Life Technologies), epidermal growth factor (final medium concentration: $20 \mathrm{ng} / \mathrm{ml}$, Peprotech), basal fibroblast growth factor (final medium concentration: $10 \mathrm{ng} / \mathrm{ml}$, Peprotech) and leukemia inhibitory factor (final medium concentration: $10 \mathrm{ng} / \mathrm{ml}$, Miltenyi Biotec) and containing $37 \mathrm{mg}$ of L-glutamine, B27 supplement (2\%, Life Technologies) and N2 supplement ( $1 \%$, Life Technologies) penicillin-streptomycin ( $1 \%$, Life Technologies), Fungizone $(0.1 \%$, Life Technologies) and gentamicin $(0.1 \%$, Life Technologies) sterilize through a $0.22-\mu \mathrm{m}$ pore filter into a sterile container and stored at $4^{\circ} \mathrm{C}$. The CSCs growth medium was supplemented with 10\% ESQ-FBS (Life Technologies). Cells were maintained at $37^{\circ} \mathrm{C}$ in ambient $\mathrm{O} 2(21 \%)$ and $5 \% \mathrm{CO} 2$. Media were replenished every 48 hours and cells were passaged at a 1:4 ratio.

\section{Cardiac Differentiation Protocol in vitro}

Page $12 / 34$ 
Cloned CSCs were placed in bacteriological dishes for 4 days for cardiospheres generation in CSC growth medium. Cardiospheres were then switched to base differentiation medium consisting of StemProß-34 SFM (a serum-free medium conditioned with StemPro®-Nutrient Supplement, Gibco, Life Technologies), Ascorbic Acid (0,5 Mm, Sigma), 1-thioglycerol (4,5x10-4 M, Sigma), L-glutamine (2mM), Non-Essential Amino Acids (Gibco, Life Technologies) and penicillin-streptomycin (1\%, Life Technologies). For specific myocyte differentiation BMP-4 (10ng/ml, Peprotech), Activin-A $(50 \mathrm{ng} / \mathrm{ml}$ first day and then $10 \mathrm{ng} / \mathrm{ml}$, Peprotech), $\beta$-FGF (10ng/ml, Peprotech), Wnt-11 (150ng/ml, R\&D System) and Wnt-5a (150ng/ml, R\&D System) were added to base differentiation medium from day 4 to day 8 . Then, differentiating cardiospheres were pelleted and transferred to laminin $(1 \mu \mathrm{g} / \mathrm{ml})$ coated dishes and Dkk-1 $(150 \mathrm{ng} / \mathrm{ml}$, R\&D System) was added to base differentiation medium from day 8 to day 14 . Differentiated cardiospheres were either trypsinized for RNA isolation or fixed with 4\% PFA.

\section{Mouse Cardiomyocytes Isolation}

Cardiomyocytes were isolated from fetal, neonatal and adult C57BL/6J mouse hearts by enzymatic dissociation. Embryonic hearts were isolated from embryos mice using GentleMACS Dissociator and manufacture instructions were followed. Hearts obtained from neonatal mice were placed in a plate containing MEM/Trypsin $(20 \mu \mathrm{g} / \mathrm{ml})$ implemented with a solution containing $0.1 \%$ DNAse l, gentamicin and fungizone $(2 \mu \mathrm{l} / \mathrm{ml})$, penicillin-streptomycin $(1 \%)$, then cut into small pieces and gently triturated with a scissor. The plate was transferred on a stirrer at $37^{\circ} \mathrm{C}$ for $10^{\prime}$ and then the solution was leave to deposit for some seconds. The process has been repeated for several times. Adult hearts were excised, the aorta cannulated and hung on a retrograde perfusion system (Langendorff method), then perfused with enzyme-containing solutions. A calcium-free solution prepared as following was used to digest the hearts: sodium chloride at a final concentration of $126 \mathrm{mM}$; glucose at a final concentration of $22 \mathrm{mM}$; HEPES at a final concentration of $24 \mathrm{mM}$; potassium chloride at a final concentration of $4.4 \mathrm{mM}$; magnesium dichloride at a final concentration of $5 \mathrm{mM}$; creatine at a final concentration of $5 \mathrm{mM}$; taurine at a final concentration of $20 \mathrm{mM}$; sodium pyruvate at a final concentration of $5 \mathrm{mM}$ and Sodium dihydrogen phosphate at a final concentration of $1 \mathrm{mM}$. To increase the myocytes yield 2,3-butanedione monoxime $10 \mathrm{mM}$ was added to the solution. The solution was filtered through a $0.22-\mu \mathrm{m}$-pore filter into a sterile container and stored at $4^{\circ} \mathrm{C}$ for up to 1 week. For heart isolation a total amount of $50 \mathrm{ml}$ of buffer was used. Briefly, the hearts were first perfused with the calcium-free solution, followed by type II collagenase digestion in presence of $\mathrm{Ca}^{2+} 0.1 \mathrm{mM}$ and a then washed with a $\mathrm{Ca}^{2+} 0.1 \mathrm{mM}$ solution. When

perfusion was completed, the hearts were taken down from the cannula, cuts into small pieces and gently triturated with a pipette. To enrich the cell suspension with viable cardiac myocytes and to remove large or undigested tissue preparations were filtered through a $100 \mu \mathrm{m}$ cell strainer and then centrifuge to separate cardiomyocytes cells from other cell types present in the heart. Viable cardiomyocytes were then allowed to sediment by gravity. The sedimentation was normally repeated several times. Rod shaped cardiac myocytes were incubated for 45 minutes at $37^{\circ} \mathrm{C}$ in ambient $\mathrm{O}_{2}(21 \%)$ and $5 \% \mathrm{CO}_{2}$ in DMEM medium (Sigma) supplemented with 10\% FBS (Life Technologies) and then used for experimental purpose. 


\section{Quantitative RT-PCR (qRT-PCR)}

RNA was extracted from CSCs, iCMs and aCM, using TRIzol Reagent (Ambion) and quantified using a Nanodrop 2000 Spectrophotometer (Thermo Fisher Scientific). Reverse transcription was performed with 0.5-1 $\mu \mathrm{g}$ of RNA using the High Capacity cDNA Kit (Applied Biosystems). Quantitative RT-PCR was performed using TaqMan Primer/Probe sets (Applied Biosystems) using StepOne Plus real Time PCR System (Applied Biosystems). TaqMan Gene Expression Assay was used for quantification of Brachyury T, Mesp-1, Mef2c, Gata-4, Nkx2.5, Tbx-5, Myl 2, Hand2, Mhy6, Mhy7, Tnnt2, Actc1, Tnnt3, Atp2a, Pln, Ccna2, Rb1, Rbl1, Rbl2, Cnn1 and Acta2. miRNA expression was evaluated using TaqMan miRNA assays (Applied Biosystems) for mir-1 and mir-499. Data were processed by the $\Delta \mathrm{Ct}$ method using StepOne Software v2.3 and mRNA was normalized to the housekeeping gene, Gapdh. All reactions were carried out in triplicate.

\section{Immunocytochemistry}

For myogenic differentiation, cardiospheres derived from cloned CSCs, were cultured on glass chamber slides for 14 days and, after fixation with 4\% PFA for 20 min on ice, stained with rabbit anti-cardiac troponin I (1:100 dilution, Abcam) or mouse anti-MF20 (1:50 diluition, DSHB). Neonatal cardiomyocytes and adult cardiomyocytes were fixed with $4 \%$ PFA for 20 min on ice, stained with rabbit anti-cardiac troponin I (1:100 dilution, Abcam). Cells were incubated with anti-rabbit-488 or anti-mouse-594 secondary antibody (1:100 dilution, Jackson Immunoresearch). Nuclei were counterstained with the DNA binding dye, 4,6-diamidino-2-phenylindole (DAPI, Sigma) at $1 \mu \mathrm{g} / \mathrm{ml}$. Fluorescence was visualized and images acquired with confocal microscopy (LEICA TCS SP8).

\section{RNA-seq}

\section{Cell Samples}

Cell samples for RNASeq analysis were obtained from cloned CSCs, iCMs and three biological replicates to archive the analysis were performed. aCMs samples were obtained from hearts isolated from adult C57/BI6 mice and two biological replicates to archive the analysis were performed.

\section{RNA extraction}

RNA was extracted from CSCs, iCMs and aCMs using TRIzol Reagent (Ambion). The extracted RNA integrity was measured with an Agilent 2100 Bioanalyzer (Agilent Technologies, Santa Clara, CA, USA), and a sample with an RNA integrity number $\geqslant 8$ was considered acceptable.

\section{Library preparation}

The libraries were generated using depleted RNA obtained from $1 \mu \mathrm{g}$ total RNA by TruSeq Sample Preparation RNA Kit (Illumina, Inc., San Diego, CA, USA) according to the manufacturer's protocol without further modifications. The first step involves the removal of ribosomal RNA (rRNA) using biotinylated, target-specific oligos combined with Ribo-Zero rRNA removal beads that depletes samples of both cytoplasmic and mitochondrial rRNA. Following purification, the RNA is fragmented into small pieces using divalent cations under elevated temperature. The cleaved RNA fragments are copied into first 
strand cDNA using reverse transcriptase and random primers, followed by second strand CDNA synthesis using DNA Polymerase I and RNase $\mathrm{H}$. These cDNA fragments then have the addition of a single ' $\mathrm{A}$ ' base and subsequent ligation of the adapter (multiple indexing adapters were ligate to the ends of the ds CDNA). The products are purified and enriched with PCR to create the final CDNA library.

\section{Sequencing}

All libraries were sequenced on the Illumina HiSeq 1000, generating 100 bp paired-end reads. The libraries were divided into two groups depending on how they were prepared.

\section{Bioinformatics analysis: mapping reads to the reference genome}

To perform RNA-seq data analysis, we used Strand NGS tools (http://www.strand-ngs.com). We set transcriptome and genome with novel splices as alignment and we use Ensembl (http://www.ensembl.org/index.html) as transcript model. Raw data sequences (that is, reads) have been pre-processed and then been related to mus musculus genome. Parameters have been set as follows: (i) minimum percentage identity $=90 \%$; (ii) maximum percentage gaps $=5 \%$; (iii) maximum number of novel splices $=1$; and (iv) minimum match length $=25$. Reads with $<5$ valid matches have been filtered out, finally obtaining expression values for reads (quantification phase). To detect novel genes and exons we set the parameters, in term of percentile, as follows: minimum exon length equals to 10.0; minimum intron length equals to 10.0; maximum intron length equals to 90.0; minimum gene length equals to 10.0; minimum exon RPKM equals to 50.0, where RPKM, stands for reads per kilo base per million reads, used to represent read counts over a region of interest.

Differential expressions were performed using DESeq algorithm, available in StrandNGS (Strand Life Sciences, Bangalore, KA, India). We performed FC analysis on filtered expression values using normalized signal values that are in log-scale. We considered a FC cut off equals to 2.5 to identify up-and downregulated genes Heat maps have been created using Gitools (http://www.gitools.org/) and a hierarchical clustering has been performed using Euclidean distance metric and complete (maximum) linkage clustering as linkage method. Enrichment analysis has been performed with Gitools importing the data from the Gene Ontology databases and relating genes to biological processes, finding significantly

enriched GO terms to be compared with the data set analysed ${ }^{94}$. Statistic values are used to select the more interesting set of genes in terms of biological processes to annotate and guide clustering analysis. To discriminate between over- and under-expressed pathways we selected to display the Z-score value.

\section{7. smallRNA-Seq}

\section{Libraries preparation}

smallRNA-Seq libraries were prepared as previously described ${ }^{35,95}$. In details, libraries were generated from 120 ng using TruSeq Small RNA Sample Prep Kits (Illumina Inc.). Libraries were sequenced in single read mode, $1 \times 50$ cycles on HiSeq 2500 (Illumina Inc.). The quality of the sequenced data was assessed using FastQC (FastQC: A Quality Control Tool for High Throughput Sequence Data) and they were trimmed using Cutadapt ${ }^{96}$ with default parameters. Subsequently, the filtered data were analyzed using 
iSMART $^{97}$. The raw counts were normalized using DESeq2 ${ }^{98}$ (version 1.28.1) on $\mathrm{R}$ (version 4.0.2). The differentially expressed miRNA were filtered with a p-adjust $<0.05$ and a Fold Change of |1.5| (Log2 FC |0.6|). Volcano plots were generated using EnhancedVolcano library on R. Circos plots were generated using Circos (version 0.69-9) ${ }^{99}$. PCA plots were created using R.

\section{Target prediction}

mRNAs targets of differentially expressed miRNAs were detected with CyTargetLinker v4.1.0 ${ }^{100}$, an open source network tool of Cytoscape, investigating mirtarbase v8.0 and Targetscan v7.2 databases.

\section{Functional Annotation}

Functional and pathway analysis were performed using Gene Ontology [BiNGO (cytoscape 2.8.0)] Only selected functions and pathways showing a p-value $\leq 0.05$ were considered.

\section{Integrated analysis of microRNA and mRNA expression profiles}

Integrated analysis was performed on miRNAs that were significantly modulated (|FC|=5) in CSCs vs aCMs comparison. Differentially expressed miRNAs were selected and used in Cytoscape as starting nodes (circular nodes). The interaction network of miRNAs and their targets were extended from miRTarBase (validated interactions) and TargetScan (predicted interactions) using CyTargetLinker ${ }^{100}$. Each link contains two connected nodes, source (miRNA) and target (mRNA). The miRNAs and mRNAs were coloured based on changes in gene expression (green as down-regulated, red as up-regulated). We next performed, a functional analysis of all the nodes of the miRNA-mRNA target networks using Gene Ontology and GO Annotations ${ }^{101,102}$ and Panther Classification System ${ }^{103}$. Selected biological processes were imported as networks in Cytoscape, then merged for each of our extended miRNA-mRNA target networks.

\section{Lentivirus-mediated miRNA expression.}

Lentivirus-vector-overexpressing miRNAs were generated by Abmgood and contained an expression module for GFP that enabled the detection of cells with positive infection and transduction. Lentivirusmediated miRNA expression was conducted on mouse CSCs. Cells were seeded at a density of $0.5 \times 10^{5}$ cells in 12-well plates and incubate at $37^{\circ} \mathrm{C}$ with $5 \% \mathrm{CO}_{2}$ overnight. The day after, cells were infected with lentivirus at 50 multiplicity of infection (MOl) using polybrene at a concentration of $6 \mu \mathrm{g} / \mathrm{ml}$. Medium was replaced with fresh medium 24 hours after infection. Infected cells were selected for the stable expression of each lentivirus vectors using puromycin $(4 \mu \mathrm{g} / \mathrm{ml})$ for 48 hours. Quantification of miRNA was performed in two-step RT-PCR according to the TaqMan MicroRNA Assays protocol.

\section{Statistical analysis}

Data are reported as mean \pm SD. Significance between 2 groups was determined by Student's t-test and in multiple comparisons by the analysis of variance (ANOVA) using GraphPad Prism (GraphPad Software). In the event that ANOVA justified post-hoc comparisons between group means, these were corrected using Tukey's multiple-comparisons test. 


\section{Declarations}

Conflicts of Interest: "The authors declare no conflict of interest."

Acknowledgments: Nothing to acknowledge.

Funding: This research was funded by grants from the Ministry of Education, University and Research (PRIN2015 2015ZTT5KB_004, 2017NKB2N4_005), PON-AIM - 1829805-2, PON03PE00009_2-iCARE and ARS01_01226-PerMedNet (CUP: D26C18000260005) and POR Prodotti Alimentari.

\section{References}

1. Olson EN. Gene regulatory networks in the evolution and development of the heart. Science 313, 1922-1927 (2006).

2. Waardenberg AJ, Ramialison M, Bouveret R, Harvey RP. Genetic networks governing heart development. Cold Spring Harbor perspectives in medicine 4, a013839 (2014).

3. Hill JT, Demarest B, Gorsi B, Smith M, Yost HJ. Heart morphogenesis gene regulatory networks revealed by temporal expression analysis. Development 144, 3487-3498 (2017).

4. Cordes KR, Srivastava D. MicroRNA regulation of cardiovascular development. Circulation research 104, 724-732 (2009).

5. Lock MC, et al. The role of miRNA regulation in fetal cardiomyocytes, cardiac maturation and the risk of heart disease in adults. The Journal of physiology 596, 5625-5640 (2018).

6. Catalucci D, Latronico MV, Condorelli G. MicroRNAs control gene expression: importance for cardiac development and pathophysiology. Annals of the New York Academy of Sciences 1123, 20-29 (2008).

7. Condorelli G, Dimmeler S. MicroRNAs: Components of an integrated system controlling cardiac development, physiology, and disease pathogenesis.). Oxford University Press (2008).

8. Latronico MV, Catalucci D, Condorelli G. Emerging role of microRNAs in cardiovascular biology. Circulation research 101, 1225-1236 (2007).

9. Chen J-F, et al. Targeted deletion of Dicer in the heart leads to dilated cardiomyopathy and heart failure. Proceedings of the National Academy of Sciences 105, 2111-2116 (2008).

10. Zhao Y, et al. Dysregulation of cardiogenesis, cardiac conduction, and cell cycle in mice lacking miRNA-1-2. Cell 129, 303-317 (2007). 
11. Peng $Y$, et al. Critical roles of miRNA-mediated regulation of TGF $\beta$ signalling during mouse cardiogenesis. Cardiovascular research 103, 258-267 (2014).

12. Mummery CL, Zhang J, Ng ES, Elliott DA, Elefanty AG, Kamp TJ. Differentiation of human embryonic stem cells and induced pluripotent stem cells to cardiomyocytes: a methods overview. Circulation research 111, 344-358 (2012).

13. Parrotta El, Lucchino V, Scaramuzzino L, Scalise S, Cuda G. Modeling Cardiac Disease Mechanisms Using Induced Pluripotent Stem Cell-Derived Cardiomyocytes: Progress, Promises and Challenges. International Journal of Molecular Sciences 21, 4354 (2020).

14. Parrotta $\mathrm{E}$, et al. Two sides of the same coin? Unraveling subtle differences between human embryonic and induced pluripotent stem cells by Raman spectroscopy. Stem cell research \& therapy $\mathbf{8}, 1$ 12 (2017).

15. Chen J-F, et al. The role of microRNA-1 and microRNA-133 in skeletal muscle proliferation and differentiation. Nature genetics 38, 228-233 (2006).

16. Liu N, et al. microRNA-133a regulates cardiomyocyte proliferation and suppresses smooth muscle gene expression in the heart. Genes \& development 22, 3242-3254 (2008).

17. Sluijter JP, et al. MicroRNA-1 and-499 regulate differentiation and proliferation in human-derived cardiomyocyte progenitor cells. Arteriosclerosis, thrombosis, and vascular biology 30, 859-868 (2010).

18. van Rooij E, et al. A family of microRNAs encoded by myosin genes governs myosin expression and muscle performance. Developmental cell 17, 662-673 (2009).

19. Ishikawa $D$, et al. miRNome profiling of purified endoderm and mesoderm differentiated from hESCs reveals functions of miR-483-3p and miR-1263 for cell-fate decisions. Stem cell reports 9,1588 1603 (2017).

20. Mitchelson KR, Qin W-Y. Roles of the canonical myomiRs miR-1,-133 and-206 in cell development and disease. World journal of biological chemistry 6, 162 (2015).

21. Shen $\mathrm{X}$, et al. miR-322/-503 cluster is expressed in the earliest cardiac progenitor cells and drives cardiomyocyte specification. Proceedings of the National Academy of Sciences 113, 9551-9556 (2016).

22. Lee DS, et al. Defined microRNAs induce aspects of maturation in mouse and human embryonicstem-cell-derived cardiomyocytes. Cell reports 12, 1960-1967 (2015).

23. Kuppusamy KT, et al. Let-7 family of microRNA is required for maturation and adult-like metabolism in stem cell-derived cardiomyocytes. Proceedings of the National Academy of Sciences 112, E2785-E2794 (2015). 
24. Mononen MM, Leung CY, Xu J, Chien KR. Trajectory mapping of human embryonic stem cell cardiogenesis reveals lineage branch points and an ISL1 progenitor-derived cardiac fibroblast lineage. Stem Cells 38, 1267-1278 (2020).

25. Parrotta El, Scalise S, Scaramuzzino L, Cuda G. Stem Cells: The Game Changers of Human Cardiac Disease Modelling and Regenerative Medicine. International journal of molecular sciences 20 , 5760 (2019).

26. Beltrami AP, et al. Adult cardiac stem cells are multipotent and support myocardial regeneration. cell 114, 763-776 (2003).

27. Messina $\mathrm{E}$, et al. Isolation and expansion of adult cardiac stem cells from human and murine heart. Circulation research 95, 911-921 (2004).

28. Fransioli $\mathrm{J}$, et al. Evolution of the c-kit-positive cell response to pathological challenge in the myocardium. Stem cells 26, 1315-1324 (2008).

29. Smith AJ, et al. Isolation and characterization of resident endogenous c-Kit+ cardiac stem cells from the adult mouse and rat heart. Nature protocols 9, 1662 (2014).

30. Ellison GM, et al. Endogenous cardiac stem cell activation by insulin-like growth factor1/hepatocyte growth factor intracoronary injection fosters survival and regeneration of the infarcted pig heart. Journal of the American College of Cardiology 58, 977-986 (2011).

31. Linke A, et al. Stem cells in the dog heart are self-renewing, clonogenic, and multipotent and regenerate infarcted myocardium, improving cardiac function. Proceedings of the national academy of sciences 102, 8966-8971 (2005).

32. Torella D, Ellison GM, Méndez-Ferrer S, Ibanez B, Nadal-Ginard B. Resident human cardiac stem cells: role in cardiac cellular homeostasis and potential for myocardial regeneration. Nature Clinical Practice Cardiovascular Medicine 3, S8-S13 (2006).

33. Bearzi C, et al. Human cardiac stem cells. Proceedings of the National Academy of Sciences 104, 14068-14073 (2007).

34. Arsalan $\mathrm{M}$, et al. Distribution of cardiac stem cells in the human heart. International Scholarly Research Notices 2012, (2012).

35. Scalise M, et al. Atrial myxomas arise from multipotent cardiac stem cells. European heart journal 41, 4332-4345 (2020).

36. Vicinanza $\mathrm{C}$, et al. Adult cardiac stem cells are multipotent and robustly myogenic: c-kit expression is necessary but not sufficient for their identification. Cell Death \& Differentiation 24, 2101-2116 (2017). 
37. Cianflone $\mathrm{E}$, et al. Molecular basis of functional myogenic specification of Bona Fide multipotent adult cardiac stem cells. Cell Cycle 17, 927-946 (2018).

38. Scalise M, et al. Heterogeneity of Adult Cardiac Stem Cells. Stem Cells Heterogeneity in Different Organs, 141-178 (2019).

39. Marino $\mathrm{F}$, et al. Role of c-kit in myocardial regeneration and aging. Frontiers in endocrinology 10, 371 (2019).

40. Marotta $\mathrm{P}$, et al. Combining cell and gene therapy to advance cardiac regeneration. Expert opinion on biological therapy $18,409-423$ (2018).

41. Cianflone $\mathrm{E}$, et al. Targeting cardiac stem cell senescence to treat cardiac aging and disease. Cel/s 9, 1558 (2020).

42. Cianflone $\mathrm{E}$, et al. Adult cardiac stem cell aging: a reversible stochastic phenomenon? Oxidative medicine and cellular longevity 2019, (2019).

43. Aquila I, et al. The use and abuse of Cre/Lox recombination to identify adult cardiomyocyte renewal rate and origin. Pharmacological research 127, 116-128 (2018).

44. Leppo MK, Hare JM, Messina E, Giacomello A, Abraham MR, Marbán E. Regenerative potential of cardiosphere-derived cells expanded from percutaneous endomyocardial biopsy specimens. Circulation, (2007).

45. Chimenti I, et al. Isolation and expansion of adult cardiac stem/progenitor cells in the form of cardiospheres from human cardiac biopsies and murine hearts. In: Somatic Stem Cells). Springer (2012).

46. Li TS, et al. Cardiospheres recapitulate a niche-like microenvironment rich in stemness and cellmatrix interactions, rationalizing their enhanced functional potency for myocardial repair. Stem cells $\mathbf{2 8}$, 2088-2098 (2010).

47. Mancuso T, et al. Unravelling the Biology of Adult Cardiac Stem Cell-Derived Exosomes to Foster Endogenous Cardiac Regeneration and Repair. International Journal of Molecular Sciences 21, 3725 (2020).

48. Vicinanza C, et al. Kit cre knock-in mice fail to fate-map cardiac stem cells. Nature 555, E1-E5 (2018).

49. Aquila I, et al. c-kit Haploinsufficiency impairs adult cardiac stem cell growth, myogenicity and myocardial regeneration. Cell death \& disease 10, 1-19 (2019).

50. Torella $\mathrm{D}$, et al. Carbonic anhydrase activation is associated with worsened pathological remodeling in human ischemic diabetic cardiomyopathy. Journal of the American Heart Association 3, 
e000434 (2014).

51. Di Siena S, et al. Activated c-Kit receptor in the heart promotes cardiac repair and regeneration after injury. Cell death \& disease 7, e2317-e2317 (2016).

52. Torella D, Indolfi C, Nadal-Ginard B. Generation of new cardiomyocytes after injury: de novo formation from resident progenitors vs. replication of pre-existing cardiomyocytes. Annals of translational medicine 3, (2015).

53. Nadal-Ginard B, Ellison GM, Torella D. Absence of evidence is not evidence of absence: pitfalls of cre knock-ins in the c-Kit locus. Circulation research 115, 415-418 (2014).

54. Urbanek $\mathrm{K}$, et al. Intratracheal administration of mesenchymal stem cells modulates tachykinin system, suppresses airway remodeling and reduces airway hyperresponsiveness in an animal model. PLoS One 11, e0158746 (2016).

55. Van Berlo JH, et al. C-kit+ cells minimally contribute cardiomyocytes to the heart. Nature 509, 337341 (2014).

56. Neidig LE, et al. Evidence for minimal cardiogenic potential of stem cell antigen 1-positive cells in the adult mouse heart. Circulation 138, 2960-2962 (2018).

57. Wallner $\mathrm{M}$, et al. Acute catecholamine exposure causes reversible myocyte injury without cardiac regeneration. Circulation research 119, 865-879 (2016).

58. Vagnozzi RJ, Sargent MA, Lin S-CJ, Palpant NJ, Murry CE, Molkentin JD. Genetic lineage tracing of Sca-1+ cells reveals endothelial but not myogenic contribution to the murine heart. Circulation 138, 29312939 (2018).

59. Sultana $\mathrm{N}$, et al. Resident c-kit+ cells in the heart are not cardiac stem cells. Nature communications 6, 1-10 (2015).

60. Liu Q, et al. Genetic lineage tracing identifies in situ Kit-expressing cardiomyocytes. Cell research 26, 119-130 (2016).

61. Shiba Y, Hauch KD, Laflamme MA. Cardiac applications for human pluripotent stem cells. Current pharmaceutical design 15, 2791-2806 (2009).

62. Xu XQ, Soo SY, Sun W, Zweigerdt R. Global expression profile of highly enriched cardiomyocytes derived from human embryonic stem cells. Stem cells 27, 2163-2174 (2009).

63. Hong G, Zhang W, Li H, Shen X, Guo Z. Separate enrichment analysis of pathways for up-and downregulated genes. Journal of the Royal Society Interface 11, 20130950 (2014). 
64. Ridgeway AG, Wilton S, Skerjanc IS. Myocyte enhancer factor $2 \mathrm{C}$ and myogenin up-regulate each other's expression and induce the development of skeletal muscle in P19 cells. Journal of Biological Chemistry 275, 41-46 (2000).

65. Tang $X$, et al. PDGFRA gene, maternal binge drinking and obstructive heart defects. Scientific reports $8,1-7$ (2018).

66. Wystrychowski W, Patlolla B, Zhuge Y, Neofytou E, Robbins RC, Beygui RE. Multipotency and cardiomyogenic potential of human adipose-derived stem cells from epicardium, pericardium, and omentum. Stem cell research \& therapy 7, 1-12 (2016).

67. Gu W, Schneider JW, Condorelli G, Kaushal S, Mahdavi V, Nadal-Ginard B. Interaction of myogenic factors and the retinoblastoma protein mediates muscle cell commitment and differentiation. Cel/ 72, 309-324 (1993).

68. Schneider JW, Gu W, Zhu L, Mahdavi V, Nadal-Ginard B. Reversal of terminal differentiation mediated by p107 in Rb-/-muscle cells. Science 264, 1467-1471 (1994).

69. MacLellan W, et al. Overlapping roles of pocket proteins in the myocardium are unmasked by germ line deletion of p130 plus heart-specific deletion of Rb. Molecular and Cellular Biology 25, 2486 (2005).

70. Alfar EA, El-Armouche A, Guan K. MicroRNAs in cardiomyocyte differentiation and maturation.). Oxford University Press (2018).

71. Li J, Wang G, Jiang J, Zhang L, Zhou P, Ren H. MicroRNA-127-3p regulates myoblast proliferation by targeting Sept7. Biotechnology letters 42, 1633-1644 (2020).

72. Li J, et al. Dynamical expression of microRNA-127-3p in proliferating and differentiating $\mathrm{C} 2 \mathrm{C} 12$ cells. Asian-Australasian journal of animal sciences 29, 1790 (2016).

73. Zhai L, Wu R, Han W, Zhang Y, Zhu D. miR-127 enhances myogenic cell differentiation by targeting S1PR3. Cell death \& disease 8, e2707-e2707 (2017).

74. Yang D, Lutter D, Burtscher I, Uetzmann L, Theis FJ, Lickert H. miR-335 promotes mesendodermal lineage segregation and shapes a transcription factor gradient in the endoderm. Development 141, 514525 (2014).

75. Garate $X$, et al. Identification of the miRNAome of early mesoderm progenitor cells and cardiomyocytes derived from human pluripotent stem cells. Scientific reports 8, 1-14 (2018).

76. Zhen L-X, et al. MiR-301a promotes embryonic stem cell differentiation to cardiomyocytes. World journal of stem cells 11, 1130 (2019). 
77. Rangrez AY, et al. MicroRNA miR-301a is a novel cardiac regulator of Cofilin-2. PloS one 12, e0183901 (2017).

78. Callis TE, et al. MicroRNA-208a is a regulator of cardiac hypertrophy and conduction in mice. The Journal of clinical investigation 119, 2772-2786 (2009).

79. Piegari E, et al. MicroRNA-34a regulates doxorubicin-induced cardiotoxicity in rat. Oncotarget 7, $62312(2016)$.

80. Guo H, Ingolia NT, Weissman JS, Bartel DP. Mammalian microRNAs predominantly act to decrease target mRNA levels. Nature 466, 835-840 (2010).

81. Liu N, Olson EN. MicroRNA regulatory networks in cardiovascular development. Developmental cell 18, 510-525 (2010).

82. Hulliger MF, et al. An Integrative miRNA-mRNA Expression Analysis Reveals Striking Transcriptomic Similarities between Severe Equine Asthma and Specific Asthma Endotypes in Humans. Genes 11, 1143 (2020).

83. Nelson TJ, Balza Jr R, Xiao Q, Misra RP. SRF-dependent gene expression in isolated cardiomyocytes: regulation of genes involved in cardiac hypertrophy. Journal of molecular and cellular cardiology 39, 479-489 (2005).

84. Wilson KD, et al. Dynamic microRNA expression programs during cardiac differentiation of human embryonic stem cells: role for miR-499. Circulation: Cardiovascular Genetics 3, 426-435 (2010).

85. Winnier G, Blessing M, Labosky PA, Hogan B. Bone morphogenetic protein-4 is required for mesoderm formation and patterning in the mouse. Genes \& development 9, 2105-2116 (1995).

86. Zhang H, Bradley A. Mice deficient for BMP2 are nonviable and have defects in amnion/chorion and cardiac development. Development 122, 2977-2986 (1996).

87. Ueno $\mathrm{S}$, et al. Biphasic role for Wnt/ $\beta$-catenin signaling in cardiac specification in zebrafish and embryonic stem cells. Proceedings of the National Academy of Sciences 104, 9685-9690 (2007).

88. Nakamura T, Sano M, Songyang Z, Schneider MD. A Wnt-and $\beta$-catenin-dependent pathway for mammalian cardiac myogenesis. Proceedings of the National Academy of Sciences 100, 5834-5839 (2003).

89. Valente M, Nascimento DS, Cumano A, Pinto-Do-Ó P. Sca-1+ cardiac progenitor cells and heartmaking: a critical synopsis. Stem cells and development 23, 2263-2273 (2014).

90. Van Vliet $\mathrm{P}$, et al. Progenitor cells isolated from the human heart: a potential cell source for regenerative therapy. Netherlands Heart Journal 16, 163-169 (2008). 
91. Smits AM, et al. Human cardiomyocyte progenitor cells differentiate into functional mature cardiomyocytes: an in vitro model for studying human cardiac physiology and pathophysiology. Nature protocols 4, 232 (2009).

92. Wang Z, Gerstein M, Snyder M. RNA-Seq: a revolutionary tool for transcriptomics. Nature reviews genetics 10, 57-63 (2009).

93. Bishop SP, Zhou Y, Nakada Y, Zhang J. Changes in Cardiomyocyte Cell Cycle and Hypertrophic Growth During Fetal to Adult in Mammals. Journal of the American Heart Association 10, e017839 (2021).

94. Perez-Llamas C, Lopez-Bigas N. Gitools: analysis and visualisation of genomic data using interactive heat-maps. PloS one 6, e19541 (2011).

95. Torella $\mathrm{D}$, et al. miRNA regulation of the hyperproliferative phenotype of vascular smooth muscle cells in diabetes. Diabetes 67, 2554-2568 (2018).

96. Martin M. Cutadapt removes adapter sequences from high-throughput sequencing reads. EMBnet journal 17, 10-12 (2011).

97. Panero R, et al. iSmaRT: a toolkit for a comprehensive analysis of small RNA-Seq data. Bioinformatics 33, 938-940 (2017).

98. Love MI, Huber W, Anders S. Moderated estimation of fold change and dispersion for RNA-seq data with DESeq2. Genome biology 15, 1-21 (2014).

99. Krzywinski M, et al. Circos: an information aesthetic for comparative genomics. Genome research 19, 1639-1645 (2009).

100. Kutmon M, Kelder T, Mandaviya P, Evelo CT, Coort SL. CyTargetLinker: a cytoscape app to integrate regulatory interactions in network analysis. PloS one 8, e82160 (2013).

101. Ashburner M, et al. Gene ontology: tool for the unification of biology. Nature genetics $25,25-29$ (2000).

102. The Gene Ontology resource: enriching a GOld mine. Nucleic Acids Research 49, D325-D334 (2021).

103. $\mathrm{Mi} \mathrm{H}$, et al. PANTHER version 16: a revised family classification, tree-based classification tool, enhancer regions and extensive API. Nucleic Acids Research 49, D394-D403 (2021).

\section{Figures}



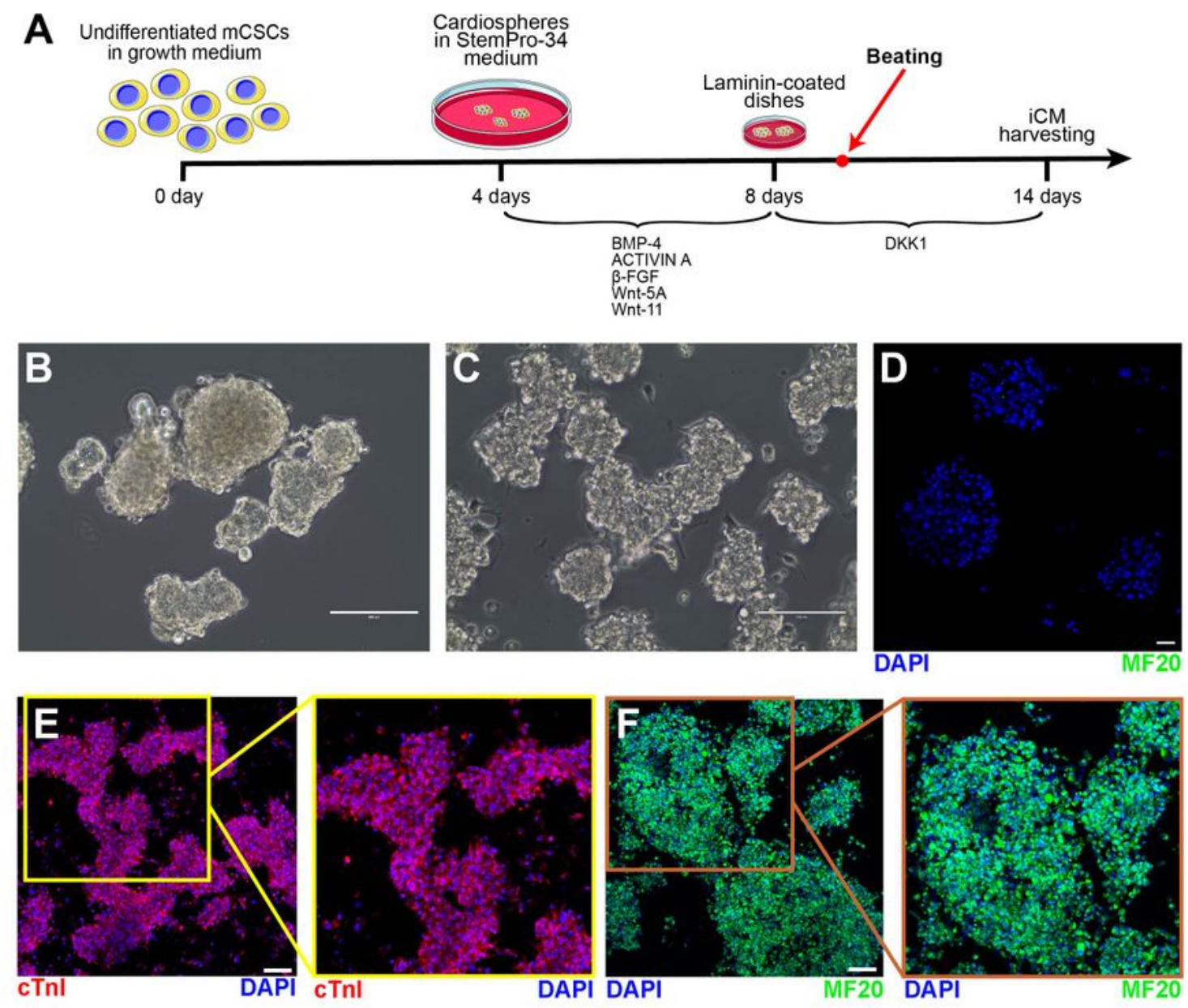

\section{$\mathbf{G}_{\text {cSC-derived iCMs }}$}
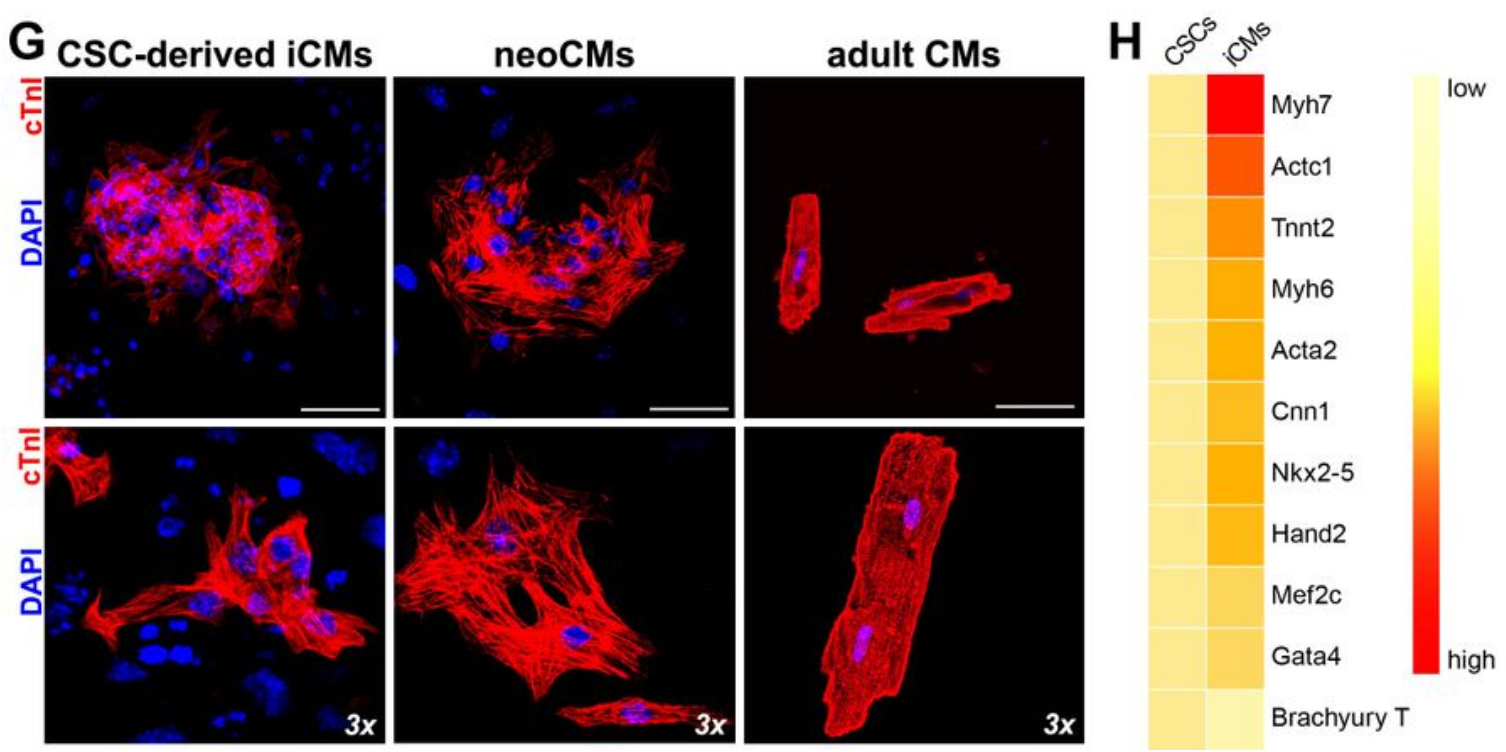

\section{Figure 1}

Cardiomyocyte differentiation potential of cloned CSCs in vitro. (A) Schematic representation shows the differentiation protocol to derive beating cardiomyocytes from cloned CSCs in vitro. (B) Representative light microscopy image of mouse cloned CSCs-derived cardiospheres (CS) 3 days before myogenic differentiation induction. Scale bar $=200 \mu \mathrm{m}$. (C) Representative light microscopy image of mouse cloned CSCs-derived cardiospheres (CS) at the end of differentiation protocol. Scale bar $=100 \mu \mathrm{m}$. (D) 
Representative confocal image of mouse cloned CSCs-derived cardiospheres (CS) 3 days before myogenic differentiation induction, showing no cTnl or MF20 expressing cells. Scale bar $=75 \mu \mathrm{m}$. $(E, F)$ Representative confocal images (areas in the squares are shown at 1.4 zoom) of functional cardiomyocytes derived from CS differentiation, showing a homogenous expression of cTnl (red) and MF20 (green). Nuclei are stained by DAPI (blue). Scale bar $=75 \mu \mathrm{m}$. (G) Confocal microscopy examples of CSC-derived iCMs, neoCMs and adult CMs labeled with cTnl (red) are shown at lower (upper panels) and higher magnification (lower panels). Similar morphology of CSC-derived iCMs and neoCMs is apparent. $(\mathrm{H})$ Heatmap showing qPCR analysis of main contractile genes in cardiosphere-derived CSCs (Actc1, Tnnt2, Myh7, Actc1, Myh6, Acta2, Cnn1) and cardiac transcription factors (Mef2c, Gata4, Nkx2.5, Hand 2 and Brachyury $\mathrm{T}$ ) after myogenic differentiation.

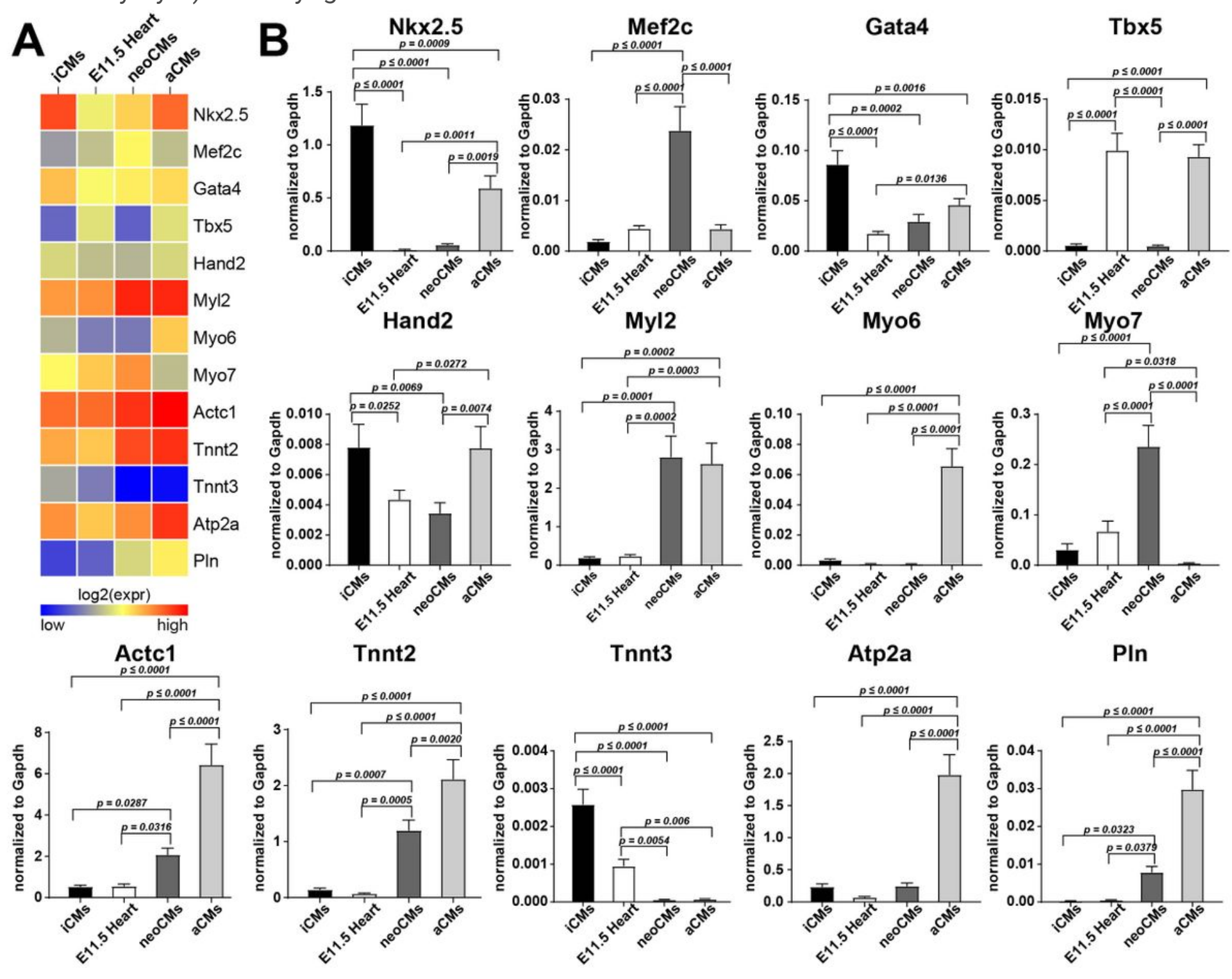

Figure 2

iCMs reach a biochemical myogenic differentiation level that is intermediate between fetal and neonatal CMs. Heatmap (A) and bar graphs (B) showing the cumulative RT-PCR data for the expression of the 
main cardiac transcription factors Gata4, Nkx2.5, Mef2c and Hand2 and the contractile genes Tnnt2, Myl2, Myh7 and Actc1 in iCMs, myocardium from 11.5 embryonic day, neoCMs and aCMs.

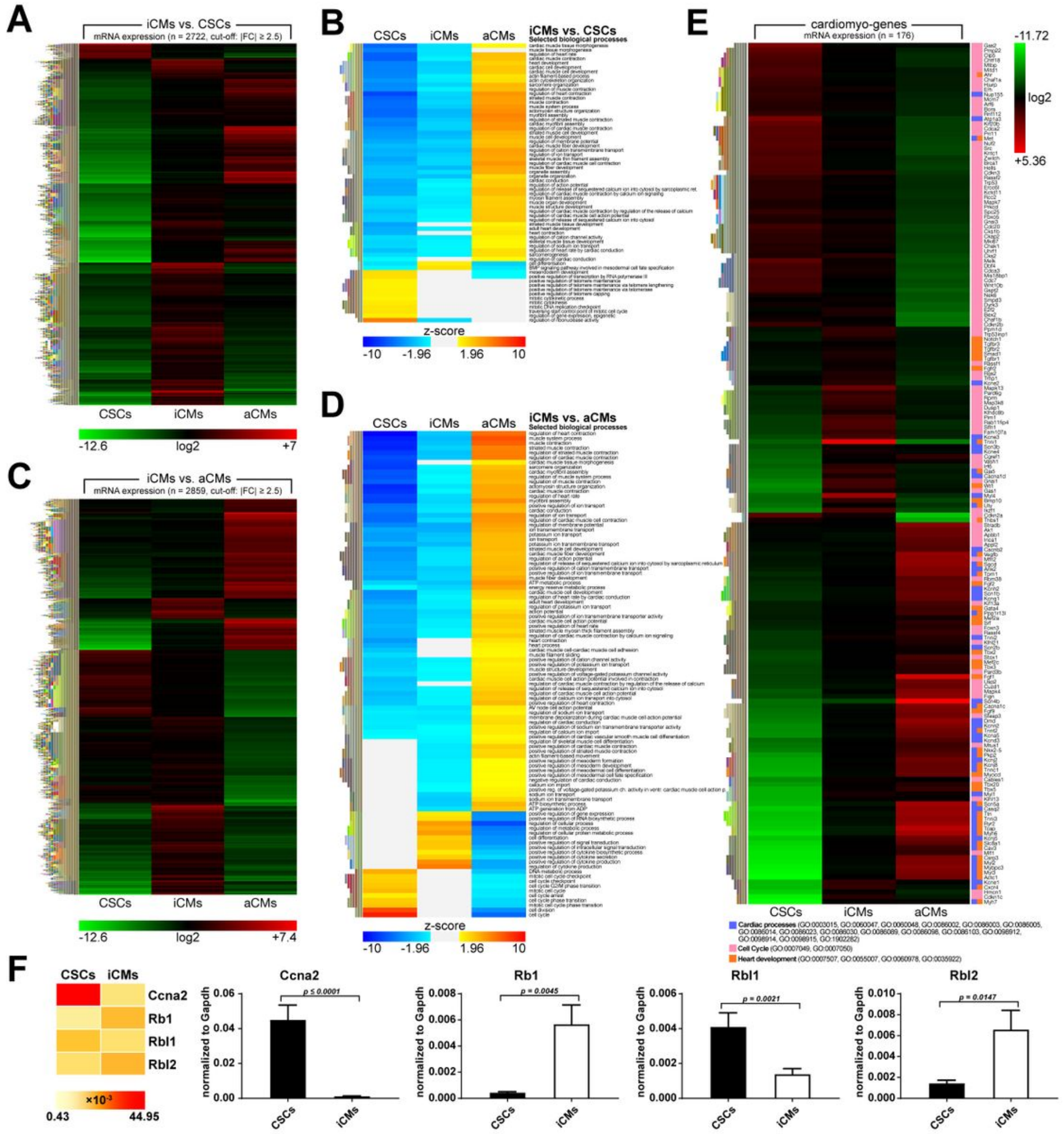

\section{Figure 3}

CSC-derived CMs (iCMs) show the typical gene expression profile of aCMs (A) Heatmap of RNAsequencing profile of iCMs vs. CSCs. The comparison identified 13491 genes expressed in both cell types, with 2601 up-regulated and 121 down-regulated genes in iCMs when compared with CSCs. (B) Heatmap 
of RNA-sequencing profile of iCMs vs. CSCs showing their enrichment analysis for specific selected biological processes. (C) Heatmap of RNA-sequencing profile of iCMs vs. aCMs. The comparison of iCMs with freshly isolated aCMs identified 13356 expressed in both cells types, with 1915 up-regulated and 944 down-regulated genes identified in iCMs when compared with aCMs. (D) Heatmap of RNA-sequencing profile of iCMs vs. aCMs showing their enrichment analysis for specific selected biological processes. (E) Heatmap of 176 selected myogenic gene expression from RNA-sequencing analysis in the aCMs vs. CSCs. (F) Heatmap and bar graphs showing the RT-PCR data for the expression of mRNA for Rb pocket proteins Rb1, Rbl1 and Rbl2 together with Cyclin A2 mRNA (Cnna2) in CSCs and iCMs. 

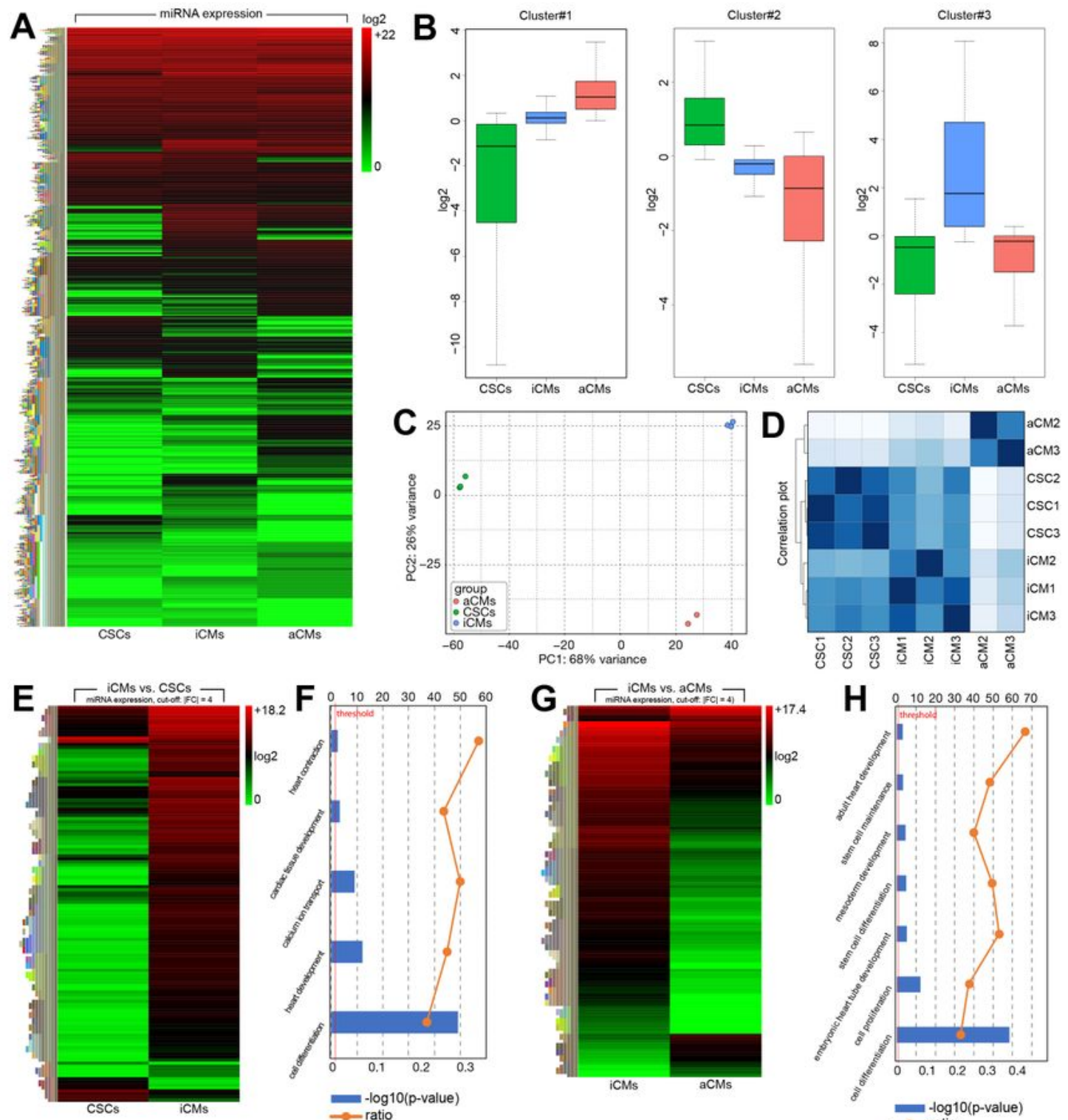

I
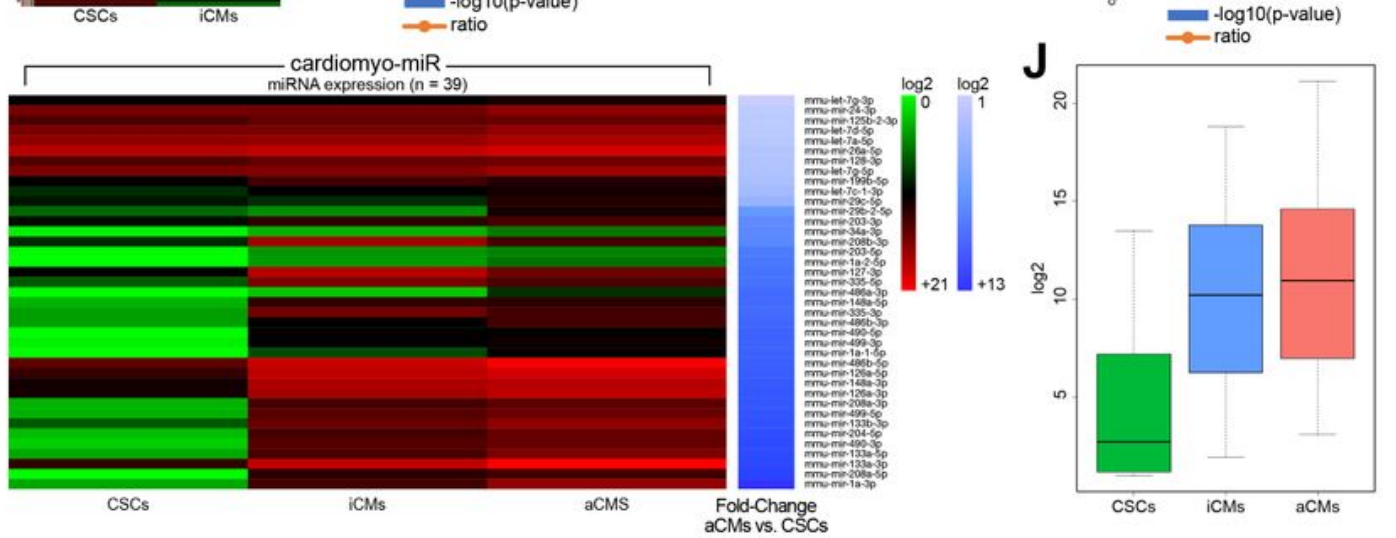

\section{Figure 4}

miRNASeq Expression of miRNAs associated with cardiac differentiation. (A) Heatmap shows miRNA expression profile obtained by RNA-Sequencing of iCMs, aCMs and CSCs. The average expression level of miRNAs in iCMs, aCMs and CSCs with a number of reads greater than a mean of 5 in at least one cell population is shown. (B) Box plots show k-means clustering of three groups of miRNAs (clusters). Each cluster includes a group of miRNAs that have the same level of expression in the three cell populations. 
(C) 2D-PCA based on the miRNA expression level applied to all the replicates of the three cell populations.

(D) Correlation plot shows differences between iCMs, aCMs and CSCs in each replicate. Higher intensity represents lower variance between the cell populations. (E) Heatmap shows differential miRNA expression profile obtained by iCMs vs. CSCs comparison. (F) Functional categorization by BinGO of differentially regulated miRNAs in iCMs vs. CSCs comparison. (G) Heatmap shows differential miRNA expression profile obtained by iCMs vs. aCMs comparison. $(\mathrm{H})$ Functional categorization by BinGO of differentially regulated miRNAs in iCMs vs. aCMs comparison. (I) Heatmap of the cardiomyo-miR expression from RNASeq analysis in the aCMs vs. CSCs comparison (with accompanying relative expression of the same miRNAs in iCMs). (J) Box plot showing the log2 normalized expression read count of the most expressed myo-miRs in aCMs compared to iCMs and CSCs samples. 


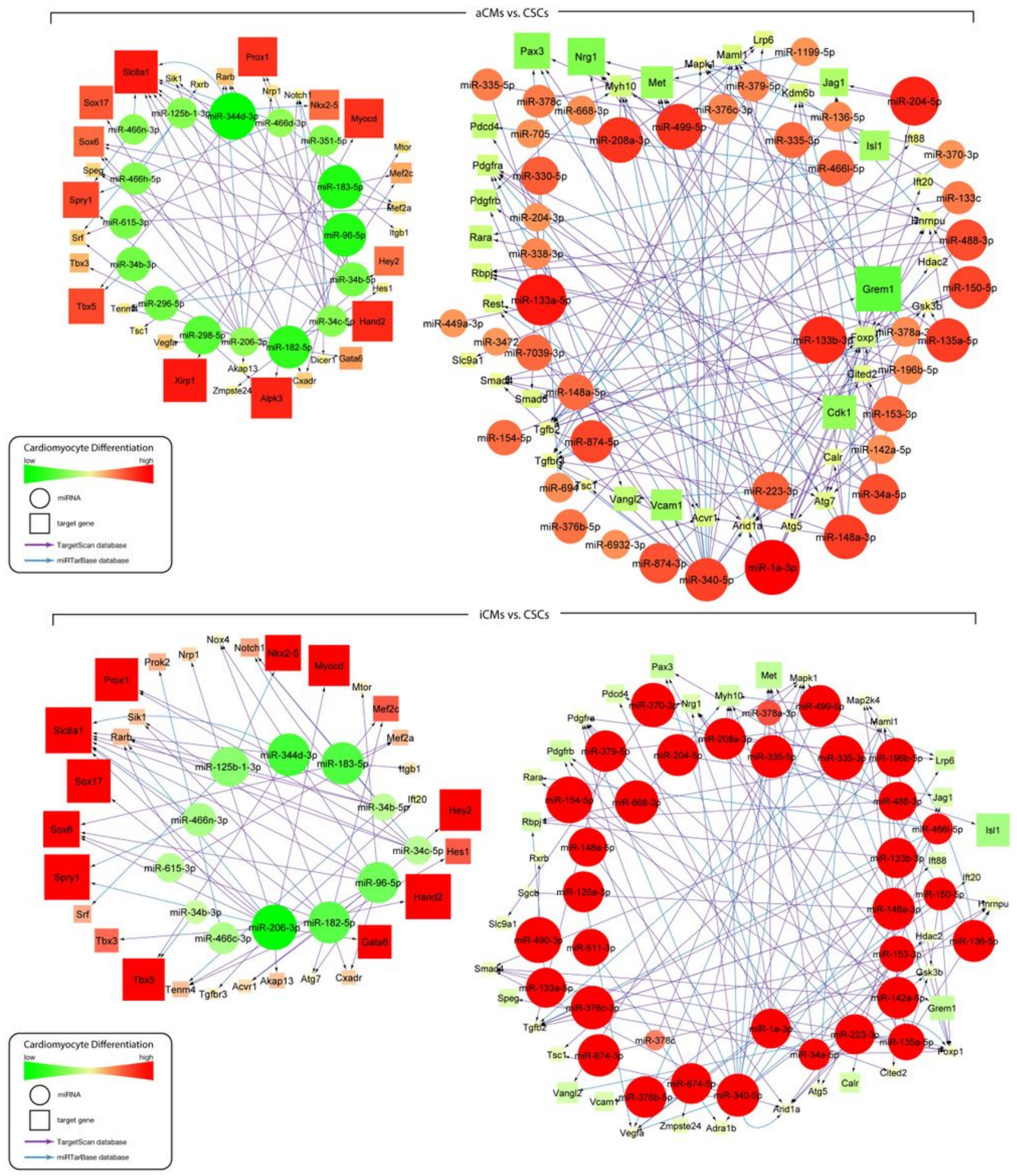

Figure 5

miRNA/mRNA networks describing the process of cardiomyocyte differentiation. The networks were built starting from the down-regulated (left) and up-regulated (right) miRNAs in the "iCMs vs. CSCs" (upper panel) and "aCMs vs. CSCs" (bottom panel) comparisons, respectively. The networks were developed inserting each miRNA/mRNA target that was effectively up-regulated for the down-regulated miRNAs and each miRNA/mRNA target that was effectively down-regulated for the up-regulated miRs. Differentially 
expressed miRNAs were used as starting nodes to generate the interaction network of miRNAs and their targets. Each link connects the source (miRNA, circle) and its targets (mRNA, squares). The colors represent changes in expression (green as down-regulated, red as up-regulated). Subsequentially functional analysis of miRNA/mRNA networks was done for selected biological processes.
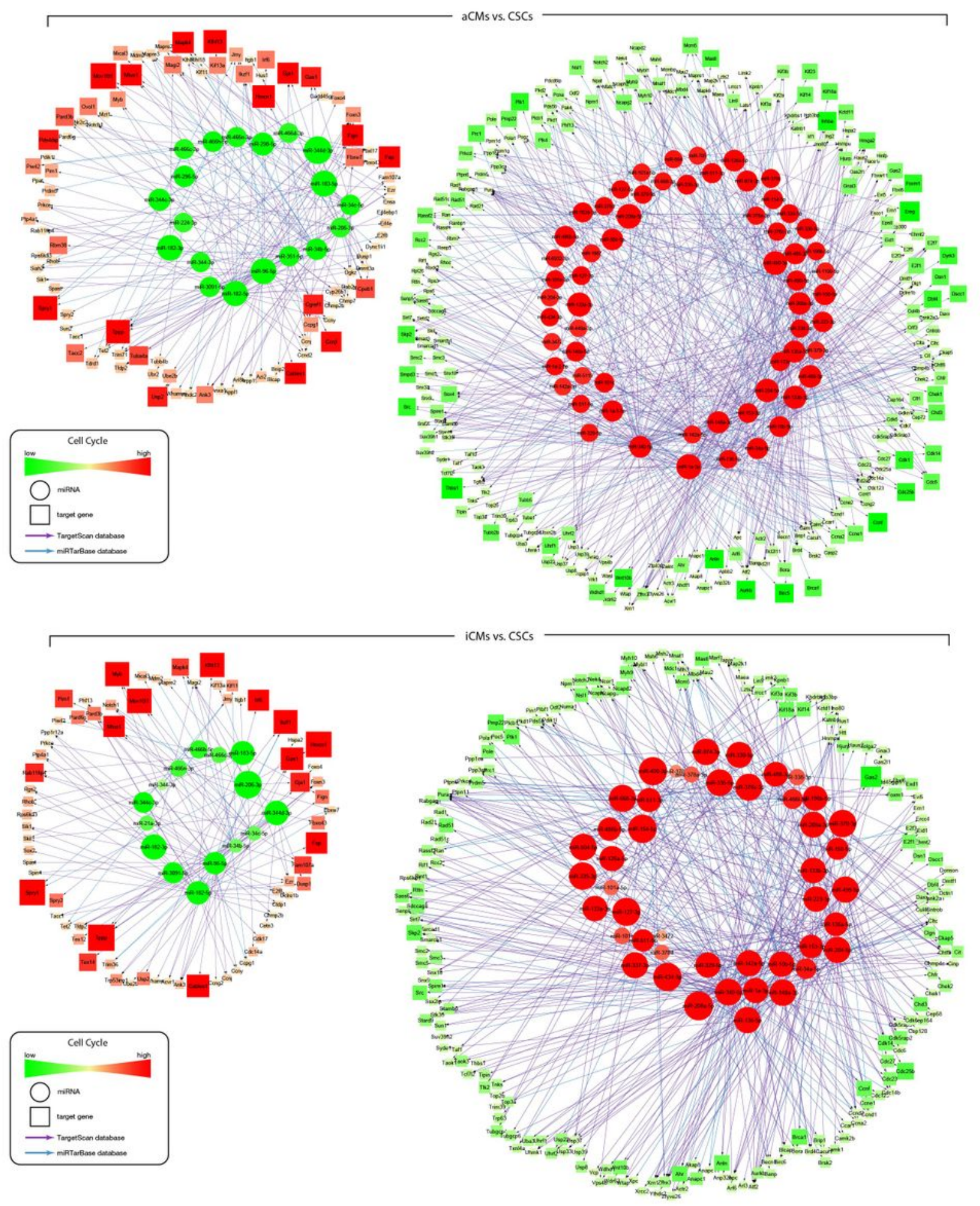

Figure 6 
miRNA/mRNA networks describing the process of cell cycle. The networks were built starting from the down-regulated (left) and up-regulated (right) miRNAs in the "aCMs vs. CSCs" (upper panel) and "iCMs vs. CSCs" (bottom panel) comparisons, respectively. The networks were developed inserting each microRNA mRNA target that was effectively up-regulated for the down-regulated miRNAs and each miRNA/mRNA target that was effectively down-regulated for the up-regulated miRNAs. Differentially expressed miRNAs were used as starting nodes to generate the interaction network of miRNAs and their targets. Each link connects the source (miRNA, circle) and its targets (mRNA, squares). The colors represent changes in expression (green as down-regulated, red as up-regulated). Subsequentially functional analysis of miRNA/mRNA networks was done for selected biological processes.

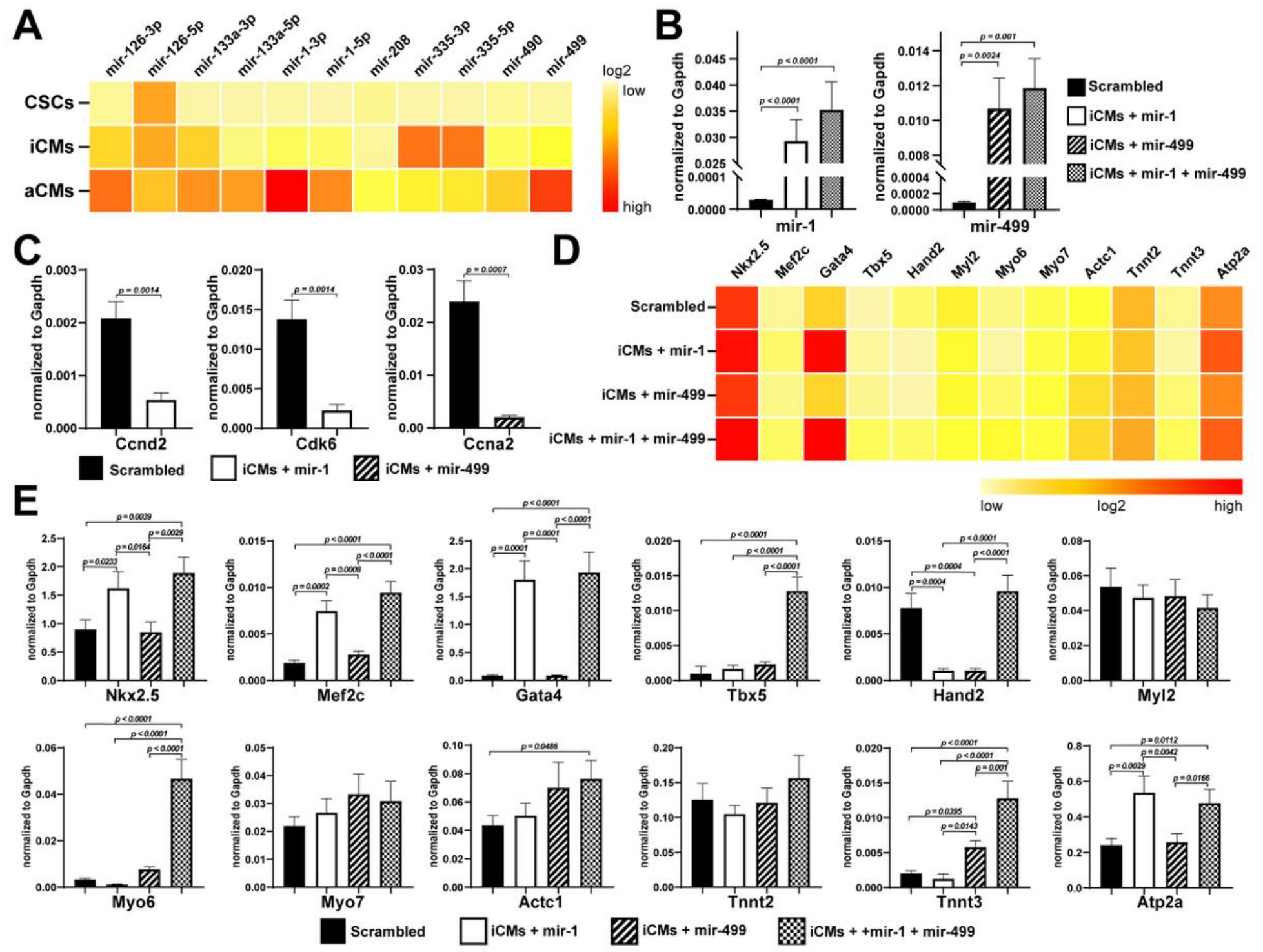

\section{Figure 7}

miR-1 and miR-499 enhanced myogenic commitment of CSC-derived iCMs in vitro.(A) Heatmap showing the upregulation of several miRNAs in aCMs. (B) Bar graphs showing the expression of miR-1 and miR499 following transduction of iCMs. (C) Bar graphs showing respectively, the expression of Cyclin D2 and Cdk6, direct targets for miR-1, and the expression of Ccna2, direct target for miR-499 in iCMs transducted 
with the respective miRNAs. (D-E) Bar graphs and heatmap showing RT-PCR data for the expression of the main cardiac transcription factors Nkx2.5, Gata4, Mef2c, Tbx5, Myl2, Myo6, Myo7, Tnnt2, Tnnt3, Actc1 and Atp2a in iCMs after tranduction of iCMs with scrambled miR, miR-1, miR-499 and miR-1+miR-499.

\section{Supplementary Files}

This is a list of supplementary files associated with this preprint. Click to download.

- SUPPLFigure1.jpg

- SUPPLFigure2.jpg

- SUPPLFigure3.jpg

- SUPPLFigure4.jpg

- SUPPLFigure5.jpg

- SupplementaryMovie1.avi

- Tables1S6.docx 